\title{
SUCCESSIVE MINIMA OF LINE BUNDLES
}

\author{
FLORIN AMBRO AND ATSUSHI ITO
}

\begin{abstract}
We introduce and study the successive minima of line bundles on proper algebraic varieties. The first (resp. last) minima are the width (resp. Seshadri constant) of the line bundle at very general points. The volume of the line bundle is equivalent to the product of the successive minima. For line bundles on toric varieties, the successive minima are equivalent to the (reciprocal of) usual successive minima of the difference of the moment polytope.
\end{abstract}

\section{INTRODUCTION}

The motivation for this paper is the classical problem in Algebraic Geometry of finding numerical criteria for a linear system on an algebraic variety to be non-empty, or to define a birational embedding. The equivalent problem in Geometry of Numbers is finding numerical criteria for a convex body to contain sufficiently many lattice points.

For a complex projective manifold $X$, Demailly [5] introduced the Seshadri constant $\epsilon(L, x)$ of an ample line bundle $L$ at a point $x$ of $X$. This invariant is constant if $x$ is very general, denoted $\epsilon(L)$ and called maximal Seshadri constant, and one can show that if $\epsilon(L)$ is sufficiently large, then the linear system $\left|K_{X}+L\right|$ is non-empty, and even defines a birational embedding (see [5, 7], or Theorem 5.1).

For a convex body $\square \subset \mathbb{R}^{d}$, the flatness theorem of Khinchin states that if the lattice width $w$ of $\square$ is sufficiently large, then $\square$ contains a lattice point (see [12]). The starting point of this paper is to observe that the flatness theorem is just a special (toric) case of Demailly's generation for adjoint line bundles in terms of maximal Seshadri constants. The key point is that on toric varieties, the maximal Seshadri constant is equivalent to the lattice width of the moment polytope:

Theorem 0.1. Let $X=T_{N} \operatorname{emb}(\Delta)$ be a proper toric variety, of dimension $d$. Let $L$ be an invariant Cartier divisor on $X$, with Seshadri constant $\epsilon$ at a general point of $X$. Let $\square_{L} \subset M_{\mathbb{R}}$ be the moment polytope, let $w$ be the lattice width of $\square_{L}$. Then

$$
\frac{w}{d} \leq \epsilon \leq w
$$

The equivalence between $w$ and $\epsilon$ not only reproves the flatness theorem, but generalizes it as follows:

Theorem 0.2. Let $\square \subset \mathbb{R}^{d}$ be a compact convex set, of lattice width $w$. If $w>d^{2}+d$, there exist $m_{0}, \ldots, m_{d} \in \mathbb{Z}^{d} \cap \operatorname{int}(\square)$ such that $m_{1}-m_{0}, \ldots, m_{d}-m_{0}$ form a basis of $\mathbb{R}^{d}$.

The proof of the equivalence involves the transference theorem of Mahler, and the fact that $w$ is the first minimum of Minkowski for the polar body $\left(\square_{L}-\square_{L}\right)^{*} \subset N_{\mathbb{R}}$. Since $\epsilon$ is proportional to the first minimum of Minkowski, we may think of $1 / \epsilon$ as a $d$-th successive minimum of $\square_{L}-\square_{L} \subset M_{\mathbb{R}}$ via the transference theorem. The question arises if the other successive minima of this 0 -symmetric body have a meaning on algebraic varieties which are not necessarily toric, and if they have a connection with Seshadri constants. The answer turns out to be positive! We introduce in this

\footnotetext{
${ }^{1} 2010$ Mathematics Subject Classification. Primary: 14C20. Secondary: 14M25.

${ }^{2}$ Keywords: Linear systems, Seshadri constants, successive minima.
} 
paper a sequence of successive minima for a line bundle on an algebraic variety, such that the last minimum coincides with the classical Seshadri constant, and in the toric case, these minima are equivalent to the (reciprocal of) successive minima of the 0-symmetric body associated to the moment polytope.

Let $X$ be a proper complex variety, let $L$ be a Cartier divisor on $X$. Let $x \in X$ be a point. For a real number $t \geq 0$, we define $\operatorname{Bs}\left|\mathcal{I}_{x}^{t+} L\right|_{\mathbb{Q}}$ to be the common zero locus of sections $s \in \Gamma(n L)(n \geq 1)$ such that $s$ vanishes at $x$ of order strictly larger than $n t$. Then $\operatorname{Bs}\left|\mathcal{I}_{x}^{t+} L\right|_{\mathbb{Q}}$ is a closed subset of $X$, increasing with respect to $t$, and equal to $X$ for $t$ sufficiently large. For integers $i \geq 1$, the $i$-th successive minimum of $L$ at $x$ is defined as

$$
\epsilon_{i}(L, x)=\inf \left\{t \geq 0 ; \operatorname{codim}_{x} \operatorname{Bs}\left|\mathcal{I}_{x}^{t+} L\right|_{\mathbb{Q}}<i\right\} .
$$

We obtain a sequence $\epsilon_{1}(L, x) \geq \epsilon_{2}(L, x) \geq \cdots \geq \epsilon_{d}(L, x) \geq 0=\epsilon_{d+1}(L, x)$, where $d=\operatorname{dim} X$. As we will see, the invariant $\epsilon_{i}(L, x)$ does not depend on a very general point $x$, is denoted $\epsilon_{i}(L)$, and called the $i$-th minimum of $L$ at a very general point. If $\kappa(L) \leq 0$, then $\epsilon_{1}(L)=0$. If $L$ has Iitaka dimension $\kappa \geq 1$, it turns outs that $\epsilon_{\kappa}(L)>0=\epsilon_{\kappa+1}(L)$. We can show that $\epsilon_{i}(L)$ are numerical invariants in case $L$ is big.

If $L$ is semiample and $x \in X$ is a smooth point, $\epsilon_{d}(L, x)$ coincides with the Seshadri constant $\epsilon(L, x)$ introduced by Demailly [5]. In particular, if $L$ is semiample, $\epsilon_{d}(L)$ coincides with the maximal Seshadri constant of $L$, usually denoted $\epsilon(L)$.

The invariant $\epsilon_{1}(L, x)$ is the largest asymptotic multiplicity that can be imposed at $x$ on sections of multiplies of $L$. It appears in the work of Nakamaye [17]. If $x \in X$ is a smooth point, it coincides with the width of $L$ at the geometric valuation induced by the exceptional divisor on the blow-up at $x$ (see [1]). Due to this property, we call $\epsilon_{1}(L, x)$ the width of $L$ at $x$. We also relate the volume of $L$ with the successive minima. By a classical argument for counting jets, we have inequalities

$$
\epsilon_{d}(L, x) \leq \sqrt[d]{\frac{\operatorname{vol}(L)}{\operatorname{mult}_{x}(X)}} \leq \epsilon_{1}(L, x) .
$$

The second main result of the paper is the analogue of Minkowski's second main theorem, namely the volume of $L$ is equivalent to the product of successive minima of $L$ at very general points:

Theorem 0.3. Let $X$ be a proper complex variety of dimension d, let $L$ be a Cartier divisor on $X$. Let $\operatorname{vol}(L)$ be the volume of $L$, let $\epsilon_{i}(L)$ be the successive minima of $L$ at very general points. Then

$$
\prod_{i=1}^{d} \epsilon_{i}(L) \leq \operatorname{vol}(L) \leq d ! \cdot \prod_{i=1}^{d} \epsilon_{i}(L) .
$$

In particular, for a big divisor $L$ on $X$ we have inequalities

$$
1 \leq \frac{\operatorname{vol}(L)}{\epsilon_{d}(L)^{d}} \leq d ! \cdot\left(\frac{\epsilon_{1}(L)}{\epsilon_{d}(L)}\right)^{d} .
$$

Understanding when the ratio $\epsilon_{1}(L) / \epsilon_{d}(L)$ is too large is an interesting problem (see Nakamaye [17]). Finally, we show that for line bundles on toric varieties, our successive minima are equivalent to the reciprocal of usual ones:

Theorem 0.4. Let $X=T_{N} \operatorname{emb}(\Delta)$ be a proper toric variety, of dimension $d$. Let $L$ be a big invariant Cartier divisor on $X$, with successive minima $\epsilon_{i}$ at a very general point. Let $\square_{L} \subset M_{\mathbb{R}}$ be the moment polytope, let $\lambda_{1}, \ldots, \lambda_{d}$ be the successive minima of the 0-symmetric convex body $\square_{L}-\square_{L} \subset M_{\mathbb{R}}$, let $\lambda_{1}^{*}, \ldots, \lambda_{d}^{*}$ be the successive minima of the polar body $\left(\square_{L}-\square_{L}\right)^{*} \subset N_{\mathbb{R}}$. Then $\epsilon_{i}, \lambda_{i}^{-1}, \lambda_{d-i+1}^{*}$ are all equivalent. More precisely,

$$
1 \leq \epsilon_{i} \cdot \lambda_{i} \leq d \cdot \frac{\epsilon_{i}}{\lambda_{d-i+1}^{*}} \leq d(d-i+1) .
$$


Our definition of successive minima is inspired by the equivalent definition of Seshadri constants due to Eckl [6, Theorem 1.1], and by the methods developed by Ein, Küchle, Lazarsfeld [7] and Nakamaye [16, 17, 18], while studying effective lower bounds for maximal Seshadri constants. Theorem 0.3, in the toric case, is just a restatement of Minkowski's second main theorem. It is implicit in the work of Nakamaye when $X$ is a surface [17, Proof of Corollary 3], and our proof is similar to his, except that we introduce certain convex polytopes in his method of counting of jets. Eventually, Theorem 0.3 reduces to a simple postulation problem in the projective space (Proposition 1.7).

A technical improvement in the study of Seshadri constants is that we no longer require positivity of the line bundles, or that the ambient space is normal. On the other hand, our invariants may not be numerical if the line bundle is not big. We hope that successive minima, and other tools from the Geometry of Numbers, may be useful in the study of adjoint linear systems.

We outline the content of this paper. In Section 1 we setup the notation, and recall basic facts about multiplicities, and linear systems. We also prove an inequality for symbolic powers of ideals in the projective space, which is elementary, but new to us. In Section 2, we define successive minima for a line bundle, study some basic properties, and compare it with Seshadri constants. In Section 3 we prove that the volume is equivalent to the product of the successive minima. In Section 4 , we estimate the successive minima for line bundles on toric varieties. In Section 5, we generalize the flatness theorem of Khinchin, as an application of known results on adjoint linear systems, plus the estimates in Section 4. In Section 6 we recall the statements of the second main theorem of Minkowski, and the transference theorem of Mahler. We also present a proof of the transference theorem in dimension two, with sharp bounds.

Acknowledgments . The first author is grateful to Max Planck Institut für Mathematik in Bonn for its hospitality and financial support. The second author was supported by Grant-in-Aid for Scientific Research 17K14162. We would like to thank the referee for useful comments and suggestions.

\section{Preliminary}

Throughout this paper, an algebraic variety $X$ is a scheme of finite type, reduced and irreducible, defined over an algebraically closed field $k$, of characteristic zero (the assumption char $k=0$ is used in Lemma 2.18 and its consequences in Section 3, and in Theorem 5.1).

Vanishing orders. Let $X$ be an algebraic variety, $\mathcal{L}$ an invertible $\mathcal{O}_{X}$-module, and $s \in \Gamma(X, \mathcal{L})$ a global section. The order of $s$ at a closed point $x \in X$, denoted $\operatorname{ord}_{x}(s)$, is the supremum after all integers $n \geq 0$ such that $s_{x} \in \mathcal{I}_{x}^{n} \cdot \mathcal{L}_{x}$. The order is $+\infty$ if $s=0$, and a non-negative integer if $s \neq 0$.

If $f: X^{\prime} \rightarrow X$ is a morphism of algebraic varieties and $f\left(x^{\prime}\right)=x$, then $f^{*} s \in \Gamma\left(X^{\prime}, f^{*} \mathcal{L}\right)$ and $\operatorname{ord}_{x^{\prime}}\left(f^{*} s\right) \geq \operatorname{ord}_{x}(s)$. In particular, if $x \in X^{\prime} \subseteq X$ is a closed subset, then $\operatorname{ord}_{x}(s) \leq \operatorname{ord}_{x}\left(\left.s\right|_{X^{\prime}}\right)$.

Suppose $X$ is smooth at the general point of a subvariety $Z \subseteq X$. The order of $s$ at $Z$ is defined as the order of $s$ at a general point of $Z$.

Order bounds. For an effective Cartier divisor $D$ on $X$, the order of $D$ at $x$, denoted $\operatorname{ord}_{x}(D)$, is defined as the order at $x$ of a local equation of $D$.

Lemma 1.1. Let $X$ be a projective algebraic variety, of dimension d, let $A$ be a very ample divisor, and $D$ an effective Cartier divisor on $X$. Then $\operatorname{ord}_{x}(D) \leq\left(D \cdot A^{d-1}\right)$ for every closed point $x \in X$.

Proof. Since $A$ is very ample, there exist $H_{1}, \ldots, H_{d-1} \in|A|$ such that $C=\cap_{i=1}^{d-1} H_{i}$ is an effective cycle passing through $x$, none of its components being contained in $D$. Then

$$
\left(D \cdot A^{d-1}\right)=(D \cdot C) \geq \operatorname{ord}_{x}\left(\left.D\right|_{C}\right) \cdot \operatorname{mult}_{x}(C) \geq \operatorname{ord}_{x}(D) \cdot \operatorname{mult}_{x}(C) \geq \operatorname{ord}_{x}(D) .
$$

We note that the first inequality follows from [10, page 233] even if $X$ is singular. 
Volume. Let $X$ be a proper algebraic variety, of dimension $d$. Let $L$ be a Cartier divisor on $X$, let $R(L)=\oplus_{n \geq 0} \Gamma\left(X, \mathcal{O}_{X}(n L)\right)$ be the induced $\mathbb{N}$-graded ring. Let $R=\oplus_{n \geq 0} R_{n} \subseteq R(L)$ be a graded subalgebra. The volume of $R$ is defined as

$$
\operatorname{vol}(R)=\limsup _{n \rightarrow \infty} \frac{\operatorname{dim}_{k} R_{n}}{n^{d} / d !} .
$$

The limsup is in fact a limit over sufficiently divisible $n$. The volume of $L$, denoted $\operatorname{vol}(L)$, is defined as the volume of $R(L)$.

We usually denote $\Gamma\left(X, \mathcal{O}_{X}(n L)\right)$ by $\Gamma(n L)$.

Iitaka map. One may define the Iitaka dimension and Iitaka map for divisors on a variety which may not be normal. These are birational invariants if the ambient space is normal.

Let $X$ be a proper variety, let $L$ be a Cartier divisor on $X$. For $n \geq 1$ such that $\Gamma(n L) \neq 0$, let $|n L|$ be the induced linear system on $X$. A basis $s_{0}, \ldots, s_{N}$ of $\Gamma(n L)$ induces a rational map $\phi_{|n L|}: X \rightarrow|n L|$, with image $X_{n}$. Define the Iitaka dimension $\kappa(L)$ of $L$ to be the maximum of $\operatorname{dim} X_{n}$ after all such $n$, and $-\infty$ if $\Gamma(n L)=0$ for all $n \geq 1$.

Suppose $\kappa(L) \geq 0$. For each $n \geq 1$ such that $\Gamma(n L) \neq 0$, let $Q_{n} \subseteq k(X)$ be the field over $k$ generated by $\left\{\frac{s^{\prime}}{s} ; s^{\prime}, s \in \Gamma(n L) \backslash 0\right\}$. The dominant rational map $\phi_{|n L|}: X \rightarrow X_{n}$ induces an isomorphism $k\left(X_{n}\right) \stackrel{\sim}{\rightarrow} Q_{n}$. The union $\cup_{|n L| \neq \emptyset} Q_{n}$ is a subfield $Q$ of $k(X)$, since $Q_{n} \cup Q_{n^{\prime}} \subseteq Q_{n+n^{\prime}}$ if $|n L|$ and $\left|n^{\prime} L\right|$ are non-empty. Since $Q$ is finitely generated over $k$, there exists an integer $m$ such that $Q_{m}=Q$. For every $n \geq 1$, we have $Q_{m}=Q_{n m}$, that is the rational maps $\phi_{|m L|}: X \rightarrow X_{m}$ and $\phi_{|n m L|}: X \rightarrow X_{n m}$ differ by a birational map $X_{n m} \rightarrow X_{m}$. We call $\phi_{|m L|}: X \rightarrow X_{m}$ the Iitaka map of $L$. The dimension of $X_{m}$ is $\kappa(L)$.

- Suppose $\kappa(L) \geq 0$ and $X$ is normal. The normalization of the graph of the litaka map $\phi_{|m L|}: X \rightarrow X_{m}$ induces a diagram

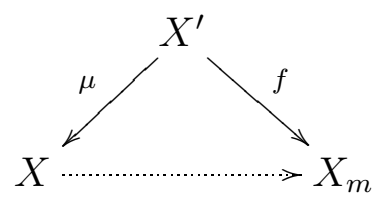

such that $X^{\prime}$ is proper and normal, $\mathcal{O}_{X}=\mu_{*} \mathcal{O}_{X^{\prime}}$, and $\kappa\left(\left.\mu^{*} L\right|_{X_{y}^{\prime}}\right)=0$ for a very general point $y \in X_{m}$. Note that $X_{y}^{\prime}$ is connected.

- Suppose $L$ is semiample, that is the linear system $|n L|$ has no base points for some $n \geq 1$. Then the Iitaka map $\phi: X \rightarrow Y$ is regular, $\mathcal{O}_{Y}=\phi_{*} \mathcal{O}_{X}$, and $n L \sim \phi^{*} A$ for some integer $n \geq 1$ and a very ample divisor $A$ on $Y$.

- Suppose $\mu: X^{\prime} \rightarrow X$ is a proper birational morphism. If $X$ and $X^{\prime}$ are normal, it follows that $\mathcal{O}_{X}=\mu_{*} \mathcal{O}_{X^{\prime}}$. In particular, $\Gamma(n L)=\Gamma\left(n \mu^{*} L\right)$ for every $n \geq 1$. Therefore the rational maps induced by non-empty linear systems $|n L|$ and $\left|n \mu^{*} L\right|$ differ by $\mu$. It follows that $\kappa(L)=\kappa\left(\mu^{*} L\right)$.

A morphism of algebraic varieties $f: X \rightarrow Y$ is called a contraction if $\mathcal{O}_{Y}=f_{*} \mathcal{O}_{X}$.

Isolated base points of linear systems. Let $X$ be a proper algebraic variety, let $L$ be a Cartier divisor on $X$ such that $\Gamma(L) \neq 0$. A basis $s_{0}, \ldots, s_{N}$ of $\Gamma(L)$ induces a rational map $\phi: X \rightarrow \mathbb{P}^{N}=$ $|L|$. Let $U=X \backslash \operatorname{Bs}|L|$, so that $\phi$ is regular on $U$. Then $\operatorname{Bs}\left|\mathcal{I}_{x}(L)\right| \cap U=\phi^{-1} \phi(x) \cap U$ for every $x \in U$.

Lemma 1.2. Let $f: X \rightarrow Y$ be a proper contraction of algebraic varieties, let $x \in X$ be a closed point such that $\operatorname{dim}_{x} X_{f(x)}=0$, where $X_{f(x)}=f^{-1} f(x)$. Then $f$ is an isomorphism over a neighborhood of $f(x)$.

Proof. By the semicontinuity of the local dimension of the fibers of $f$, there exists an open subset $U^{\prime} \ni x$ such that $\operatorname{dim}_{x^{\prime}} X_{f\left(x^{\prime}\right)}=0$ for all $x^{\prime} \in U^{\prime}$. Let $V=Y \backslash f\left(X \backslash U^{\prime}\right)$. Since $X_{f(x)}$ is 0-dimensional 
and connected, it coincides with $\{x\}$. Therefore $f(x) \in V$. Let $U=f^{-1}(V)$. Then $x \in U \subseteq U^{\prime}$. Therefore $\left.f\right|_{U}: U \rightarrow V$ is a proper contraction with finite fibers, hence a finite contraction, hence an isomorphism.

Lemma 1.3 (Lemma 5.2.24 [13]). Let $X$ be an algebraic variety. Let $\mathcal{I} \subseteq \mathcal{O}_{X}$ be an ideal sheaf, let $f: Y \rightarrow X$ be the blow-up of $X$ along $\mathcal{I}$. Let $E$ be the exceptional divisor on $Y$, defined by $\mathcal{I} \cdot \mathcal{O}_{Y}=\mathcal{O}_{Y}(-E)$. Then the natural homomorphism $\mathcal{I}^{n} \rightarrow f_{*} \mathcal{O}_{Y}(-n E)$ is an isomorphism for every $n \geq c(\mathcal{I})$, where $c(\mathcal{I}) \geq 0$ is a constant depending only on $\mathcal{I}$. Moreover, $c(\mathcal{I})=0$ if $\mathcal{I}$ is the ideal of a smooth point of $X$.

Proof. The statement is local, so we may suppose $X=\operatorname{Spec} R$ and $Y=\operatorname{Proj} S$, where $S$ is the $R$-graded ring $\oplus_{n \geq 0} I^{n}$, for some ideal $I \subseteq R$. Then $\mathcal{O}_{Y}(-n E)=\mathcal{O}_{Y}(n)=S(n)$. The natural homomorphism $S \rightarrow \oplus_{n \geq 0} \Gamma(Y, S \tilde{S}(n))$ is an isomorphism in degrees $n \geq c(I)$. This gives the claim.

If $\mathcal{I}$ is the ideal of a smooth point of $X$, then $\mathcal{I}$ is generated by a regular sequence. One checks then that $c(\mathcal{I})=0$.

Lemma 1.4 (Proposition 1.1 [8]). Let $X$ be a proper algebraic variety, let $L$ be a Cartier divisor on $X$ and $x \in X$ a closed point such that $\mathrm{Bs}|L| \cap U \subseteq\{x\}$ for some open neighborhood $U$ of $x$ in $X$. Then $x \notin \mathrm{Bs}|n L|$ for $n \gg 0$.

Proof. Let $Z$ be the base locus subscheme of $|L|$ outside $x$ (possibly empty). Let $f: Y \rightarrow X$ be the blow-up of $X$ along $\mathcal{I}_{Z}$. Let $\mathcal{I}_{Z} \cdot \mathcal{O}_{Y}=\mathcal{O}_{Y}(-E)$, so that $E$ is the exceptional divisor. Since $f$ is an isomorphism over a neighborhood of $x$, we may identify $x$ with a point of $Y$.

A basis of $\Gamma\left(X, \mathcal{I}_{Z}(L)\right)=\Gamma\left(X, \mathcal{O}_{X}(L)\right)$ induces sections of $\Gamma\left(Y, \mathcal{O}_{Y}\left(f^{*} L-E\right)\right)$ whose common zero locus is away from $E$. Therefore Bs $\left|f^{*} L-E\right| \subseteq\{x\}$. By [9, Theorem 1.10], $x \notin \operatorname{Bs}\left|n\left(f^{*} L-E\right)\right|$ for $n \geq n_{0}$. By Lemma 1.3, we may identify $\Gamma\left(X, \mathcal{I}_{Z}^{n}(n L)\right)=\Gamma\left(Y, \mathcal{O}_{Y}\left(n f^{*} L-n E\right)\right)$ for every $n \geq c\left(\mathcal{I}_{Z}\right)$. Therefore $x \notin \operatorname{Bs}\left|\mathcal{I}_{Z}^{n}(n L)\right|$ for $n \geq \max \left(n_{0}, c\left(\mathcal{I}_{Z}\right)\right)$. Since $x \notin Z$, we obtain $x \notin \operatorname{Bs}|n L|$ for $n \gg 0$.

Lemma 1.5 (Ample reduction). Let $X$ be proper, $L$ a Cartier divisor and $x \in X$ a closed point such that $\operatorname{Bs}\left|\mathcal{I}_{x}(L)\right|=\{x\}$ near $x$. Let $Z$ be the base locus subscheme of $|L|$, away from $x$ (possibly empty). Let $f: X^{\prime} \rightarrow X$ be the blow-up of $X$ along $\mathcal{I}_{Z}$, with exceptional divisor $E$. Then there exists a proper contraction $g: X^{\prime} \rightarrow Y$ to a projective algebraic variety $Y$, such that the following properties hold:

a) $f^{*} L-E \sim g^{*} A$ for some ample Cartier divisor $A$ on $Y$, and

b) $f$ is an isomorphism over a neighborhood of $x$, and $g$ is an isomorphism over a neighborhood of $g(x)$.

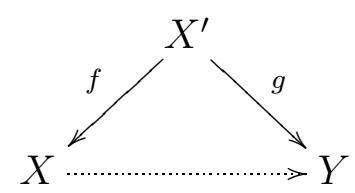

We obtain natural homomorphisms $R(L) \supseteq \oplus_{n \geq 0} \Gamma\left(\mathcal{I}_{Z}^{n}(n L)\right) \stackrel{\alpha}{\rightarrow} R\left(f^{*} L-E\right) \simeq R(A)$, and $\alpha$ is an isomorphism in sufficiently large degrees.

Proof. By construction, $\left|f^{*} L-E\right|$ is base point free near $E$. Therefore Bs $\left|f^{*} L-E\right| \subseteq\{x\}$. By the proof of Lemma 1.4, $\left|n\left(f^{*} L-E\right)\right|$ is base point free for $n \gg 0$. The Iitaka map induces the contraction $g$, with property a). For b), it is clear that $f$ is an isomorphism over a neighborhood of $x$. We identify $x$ with a point of $X^{\prime}$. Since Bs $\left|\mathcal{I}_{x}(L)\right|=\{x\}$ near $x$, we obtain $\operatorname{Bs}\left|\mathcal{I}_{x}\left(f^{*} L-E\right)\right|=\{x\}$ near $x$. Therefore $\operatorname{Bs}\left|\mathcal{I}_{x}\left(n f^{*} L-n E\right)\right|=\{x\}$ near $x$, for all $n \geq 1$. Therefore $g^{-1} g(x)=\{x\}$ near $x$. By Lemma 1.2, $g$ is an isomorphism over a neighborhood of $g(x)$. Finally, $\alpha_{n}$ is an isomorphism for $n \geq c\left(\mathcal{I}_{Z}\right)$. 
Generation of jets. Let $X$ be an algebraic variety, $L$ a Cartier divisor on $X, x \in X$ a closed point, and $p \geq 0$ an integer. We say that $L$ generates $p$-jets at $x$ if $\Gamma(L) \rightarrow \mathcal{O}_{x} / \mathcal{I}_{x}^{p+1}$ is surjective. By induction and the snake lemma, this is equivalent to the following property: the $\mathcal{O}_{X}$-module $\mathcal{I}_{x}^{n}(L)$ is generated by global sections at $x$, for every integer $0 \leq n \leq p$.

Lemma 1.6. Let $f: Y \rightarrow X$ be the blow-up at $x$, with exceptional divisor E. Suppose $\mathcal{I}_{x}^{p+1} \stackrel{\sim}{\rightarrow} f_{*}\left(\mathcal{I}_{E}^{p+1}\right)$ and $R^{1} f_{*}\left(\mathcal{I}_{E}^{p+1}\right)=0$. If $\Gamma\left(f^{*} L\right) \rightarrow \Gamma\left(\mathcal{O}_{(p+1) E}\right)$ is surjective, then $L$ generates $p$-jets at $x$. The converse holds if the natural homomorphism $H^{1}(L) \rightarrow H^{1}\left(f_{*} \mathcal{O}_{Y}(L)\right)$ is injective.

Proof. Consider the commutative diagram

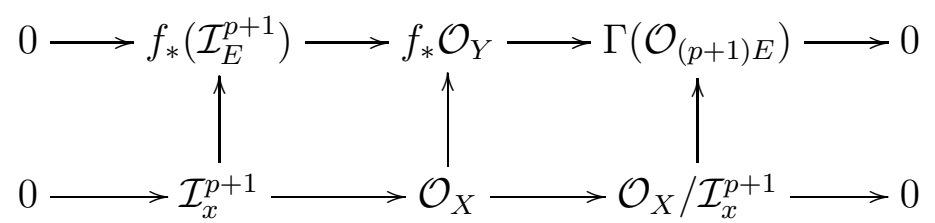

The bottom row is exact. The top is also exact, since $R^{1} f_{*}\left(\mathcal{I}_{E}^{p+1}\right)=0$. The first vertical arrow is an isomorphism, by assumption. The second vertical arrow is injective. Therefore the third arrow is injective as well. We deduce that the second and third vertical arrows have isomorphic cokernels, denoted by $\mathcal{C}$. Ignoring the first vertical arrow, tensoring with $\mathcal{O}_{X}(L)$ and passing to global sections on $X$, we obtain a commutative diagram

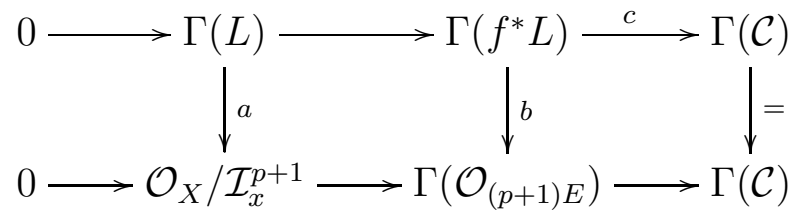

with exact rows. If $b$ is surjective, then $a$ is surjective. The converse holds if $c$ is surjective. From the exact sequence

$$
0 \rightarrow \Gamma(L) \rightarrow \Gamma\left(f_{*} \mathcal{O}_{Y}(L)\right) \stackrel{c}{\rightarrow} \Gamma(\mathcal{C}) \rightarrow H^{1}(L) \rightarrow H^{1}\left(f_{*} \mathcal{O}_{Y}(L)\right)
$$

we see that $c$ is surjective if and only if $H^{1}(L) \rightarrow H^{1}\left(f_{*} \mathcal{O}_{Y}(L)\right)$ is injective.

If $x$ is a smooth point, then $\mathcal{I}_{x}^{p+1} \stackrel{\sim}{\rightarrow} f_{*}\left(\mathcal{I}_{E}^{p+1}\right)$ and $R^{1} f_{*}\left(\mathcal{I}_{x}^{p+1}\right)=0$ for all $p \geq 0$, and $\mathcal{O}_{X}=f_{*} \mathcal{O}_{Y}$. Therefore $L$ generates $p$-jets at $x$ if and only if $\Gamma\left(f^{*} L\right) \rightarrow \Gamma\left(\mathcal{O}_{(p+1) E}\right)$ is surjective.

Postulation. For an integer $p \geq 0$, denote $\mathbb{N}^{d}(p)=\left\{\left(\alpha_{1}, \ldots, \alpha_{d}\right) \in \mathbb{N}^{d} ; \sum_{i=1}^{d} \alpha_{i}=p\right\}$. For a finite set $A$, denote by $|A|$ its cardinality. We will need the following property on the symbolic powers of ideals in the projective space.

Proposition 1.7. Let $Z_{i} \subset \mathbb{P}^{d}$ be an irreducible subvariety of codimension $i$, for $1 \leq i \leq d$. Let $p_{1}, \ldots, p_{d}, q \geq 0$ be integers. Then

$$
h^{0}\left(\mathbb{P}^{d}, \cap_{i=1}^{d} \mathcal{I}_{Z_{i}}^{\left(p_{i}\right)} \otimes \mathcal{O}_{\mathbb{P}^{d}}(q)\right) \leq\left|\cap_{i=1}^{d}\left\{\alpha \in \mathbb{N}^{d+1}(q) ; \alpha_{i}+\cdots+\alpha_{d} \leq q-p_{i}\right\}\right|
$$

When $Z_{1} \supset Z_{2} \supset \cdots \supset Z_{d}$ is a linear flag in $\mathbb{P}^{d}$, the equality holds.

Proof. 1) Suppose $Z_{1} \supset Z_{2} \supset \cdots \supset Z_{d}$ is a linear flag in $\mathbb{P}^{d}$. Choose coordinates $\left[z_{0}: \cdots: z_{d}\right]$ on $\mathbb{P}^{d}$ such that $I_{Z_{i}}=\left(z_{0}, \ldots, z_{i-1}\right)$ for all $i$. Then $\Gamma\left(\mathbb{P}^{d}, \cap_{i=1}^{d} \mathcal{I}_{Z_{i}}^{\left(p_{i}\right)} \otimes \mathcal{O}_{\mathbb{P}^{d}}(q)\right)$ is the $k$-vector space with monomial basis $z_{0}^{\alpha_{0}} \cdots z_{d}^{\alpha_{d}}$, where $\alpha \in \mathbb{N}^{d+1}(q)$ such that $\alpha_{0}+\cdots+\alpha_{i-1} \geq p_{i}$ for all $i$. Equivalently, $\alpha_{i}+\cdots+\alpha_{d} \leq q-p_{i}$ for all $i$. Therefore the inequality becomes an equality if $Z$ • is a linear flag. 
2) If $d=1$, the equality holds from 1). Suppose $d>1$, and assume by induction that the inequality holds in smaller dimension. Let $H:\{\lambda=0\}$ be a general hyperplane with respect to $Z_{\bullet}$. In particular, $H$ does not contain the point $Z_{d}$. The short exact sequence

$$
0 \rightarrow \Gamma\left(\mathbb{P}^{d}, \mathcal{O}_{\mathbb{P}^{d}}(q-1)\right) \stackrel{\otimes \lambda}{\rightarrow} \Gamma\left(\mathbb{P}^{d}, \mathcal{O}_{\mathbb{P}^{d}}(q)\right) \rightarrow \Gamma\left(H, \mathcal{O}_{H}(q)\right) \rightarrow 0
$$

induces an exact sequence

$$
0 \rightarrow \Gamma\left(\mathbb{P}^{d}, \cap_{i=1}^{d} \mathcal{I}_{Z_{i}}^{\left(p_{i}\right)} \otimes \mathcal{O}_{\mathbb{P}^{d}}(q-1)\right) \stackrel{\otimes \lambda}{\rightarrow} \Gamma\left(\mathbb{P}^{d}, \cap_{i=1}^{d} \mathcal{I}_{Z_{i}}^{\left(p_{i}\right)} \otimes \mathcal{O}_{\mathbb{P}^{d}}(q)\right) \rightarrow \Gamma\left(H, \cap_{i=1}^{d-1} \mathcal{I}_{Z_{i} \cap H}^{\left(p_{i}\right)} \otimes \mathcal{O}_{H}(q)\right) .
$$

Indeed, $\Gamma\left(\mathbb{P}^{d}, \cap_{i=1}^{d} \mathcal{I}_{Z_{i}}^{\left(p_{i}\right)} \otimes \mathcal{O}_{\mathbb{P}^{d}}(q)\right)$ consists of homogenous polynomials $F$ of degree $q$ such that $\operatorname{ord}_{x}(F) \geq p_{i}$ for every $x \in Z_{i}$, for all $i$. Then $\operatorname{ord}_{x}\left(\left.F\right|_{H}\right) \geq p_{i}$ for every $x \in Z_{i} \cap H$. If $\left.F\right|_{H}=0$, then $F=\lambda F^{\prime}$ for some homogeneous polynomial $F^{\prime}$ of degree $q-1$. For $x \in Z_{i} \backslash H$ we have $\operatorname{ord}_{x}(F)=\operatorname{ord}_{x}\left(F^{\prime}\right)$. Then $F^{\prime}$ vanishes to order at least $p_{i}$ at a general point of $Z_{i}$, hence everywhere on $Z_{i}$.

Moreover, the above exact sequence extends to a short exact sequence if $Z \bullet$ is a linear flag:

$$
0 \rightarrow \Gamma\left(\mathbb{P}^{d}, \cap_{i=1}^{d} \mathcal{I}_{Z_{i}}^{\left(p_{i}\right)} \otimes \mathcal{O}_{\mathbb{P}^{d}}(q-1)\right) \stackrel{\otimes \lambda}{\rightarrow} \Gamma\left(\mathbb{P}^{d}, \cap_{i=1}^{d} \mathcal{I}_{Z_{i}}^{\left(p_{i}\right)} \otimes \mathcal{O}_{\mathbb{P}^{d}}(q)\right) \rightarrow \Gamma\left(H, \cap_{i=1}^{d-1} \mathcal{I}_{Z_{i} \cap H}^{\left(p_{i}\right)} \otimes \mathcal{O}_{H}(q)\right) \rightarrow 0 .
$$

Indeed, we may choose coordinates as in 1), and suppose $\lambda=z_{d}$. The part $\alpha_{d} \geq 1$ of the set

$$
\cap_{i=1}^{d}\left\{\alpha \in \mathbb{N}^{d+1}(q) ; \alpha_{i}+\cdots+\alpha_{d} \leq q-p_{i}\right\}
$$

corresponds to a monomial basis of $\Gamma\left(\mathbb{P}^{d}, \cap_{i=1}^{d} \mathcal{I}_{Z_{i}}^{\left(p_{i}\right)} \otimes \mathcal{O}_{\mathbb{P}^{d}}(q-1)\right)$, and the part $\alpha_{d}=0$ corresponds to a monomial basis of $\Gamma\left(H, \cap_{i=1}^{d-1} \mathcal{I}_{Z_{i} \cap H}^{\left(p_{i}\right)} \otimes \mathcal{O}_{H}(q)\right)$. Since the dimensions add up, the sequence is exact to the right as well.

Denote $f(q)=h^{0}\left(\mathbb{P}^{d}, \cap_{i=1}^{d} \mathcal{I}_{Z_{i}}^{\left(p_{i}\right)} \otimes \mathcal{O}_{\mathbb{P}^{d}}(q)\right)$. For each $1 \leq i \leq d-1$, choose an irreducible subvariety $W_{i} \subseteq Z_{i} \cap H$, of codimension $i$ in $H \simeq \mathbb{P}^{d-1}$. The exact sequence gives

$$
f(q)-f(q-1) \leq h^{0}\left(\mathbb{P}^{d-1}, \cap_{i=1}^{d-1} \mathcal{I}_{W_{i}}^{\left(p_{i}\right)}(q)\right) .
$$

Denote $g(q)=h^{0}\left(\mathbb{P}^{d}, \cap_{i=1}^{d} \mathcal{I}_{L_{i}}^{\left(p_{i}\right)} \otimes \mathcal{O}_{\mathbb{P}^{d}}(q)\right)$, where $L_{\bullet}$ is a linear flag in $\mathbb{P}^{d}$. The exact sequence gives

$$
g(q)-g(q-1)=h^{0}\left(\mathbb{P}^{d-1}, \cap_{i=1}^{d-1} \mathcal{I}_{L_{i} \cap H}^{\left(p_{i}\right)}(q)\right)
$$

The inequality in dimension $d-1$ gives

$$
h^{0}\left(\mathbb{P}^{d-1}, \cap_{i=1}^{d-1} \mathcal{I}_{W_{i}}^{\left(p_{i}\right)}(q)\right) \leq h^{0}\left(\mathbb{P}^{d-1}, \cap_{i=1}^{d-1} \mathcal{I}_{L_{i} \cap H}^{\left(p_{i}\right)}(q)\right) .
$$

We deduce

$$
f(q)-f(q-1) \leq g(q)-g(q-1) \forall q .
$$

We have $\Gamma\left(\mathbb{P}^{d}, \mathcal{I}_{Z_{d}}^{\left(p_{d}\right)}(q)\right)=0$ for $q<p_{d}$. Therefore $f(q)=g(q)=0$ for $q<p_{d}$. By increasing induction on $q \geq p_{d}$, the above inequality gives $f(q) \leq g(q)$ for all $q$.

For real numbers $t_{1}, \ldots, t_{d}$, define a compact convex set in $\mathbb{R}^{d}$ by

$$
\square\left(t_{1}, \ldots, t_{d}\right)=\cap_{i=1}^{d}\left\{x \in \mathbb{R}_{\geq 0}^{d} ; x_{i}+\cdots+x_{d} \leq t_{i}\right\} .
$$

Note that $\square\left(t_{1}, \ldots, t_{d}\right)$ is not empty if and only if $t_{1}, \ldots, t_{d} \geq 0$. If $t_{1} \geq \cdots \geq t_{d} \geq 0$, we may compute vol $\square\left(t_{1}\right)=t_{1}, 2 \operatorname{vol} \square\left(t_{1}, t_{2}\right)=2 t_{1} t_{2}-t_{2}^{2}$, and $6 \operatorname{vol} \square\left(t_{1}, t_{2}, t_{3}\right)=\overline{6} t_{1} t_{2} t_{3}-3 t_{3}^{2} t_{1}-3 t_{3} t_{2}^{2}+t_{3}^{3}$.

Lemma 1.8. Suppose $t_{i} \geq 0$ for all $i$. Then $\operatorname{vol} \square\left(t_{1}, \ldots, t_{d}\right) \leq \prod_{i=1}^{d} t_{i}$.

Proof. We have an inclusion $\square\left(t_{1}, \ldots, t_{d}\right) \subset\left[0, t_{1}\right] \times \square\left(t_{2}, \ldots, t_{d}\right)$. Therefore

$$
\operatorname{vol} \square\left(t_{1}, \ldots, t_{d}\right) \leq t_{1} \cdot \operatorname{vol} \square\left(t_{2}, \ldots, t_{d}\right) .
$$

By induction on $d$, the desired inequality holds. 
Using this convex set, we may restate Proposition 1.7 as follows:

$$
h^{0}\left(\mathbb{P}^{d}, \cap_{i=1}^{d} \mathcal{I}_{Z_{i}}^{\left(p_{i}\right)} \otimes \mathcal{O}_{\mathbb{P}^{d}}(q)\right) \leq\left|\mathbb{Z}^{d} \cap \square\left(q-p_{1}, \ldots, q-p_{d}\right)\right| .
$$

\section{Successive minima of Line Bundles}

Let $X$ be a proper algebraic variety, of dimension $d$. Let $L$ be a Cartier divisor, with induced graded ring $R=\oplus_{n \geq 0} \Gamma(n L)$. Let $x \in X$ be a closed point. For a real number $t \geq 0$, denote

$$
\operatorname{Bs}\left|\mathcal{I}_{x}^{t+} L\right|_{\mathbb{Q}}=\cap_{n \geq 1}\left\{Z(s) ; s \in R_{n}, \operatorname{ord}_{x}(s)>n t\right\} .
$$

It is a closed subset of $X$. If $t \leq t^{\prime}$, then $\operatorname{Bs}\left|\mathcal{I}_{x}^{t+} L\right|_{\mathbb{Q}} \subseteq \operatorname{Bs}\left|\mathcal{I}_{x}^{t^{\prime}+} L\right|_{\mathbb{Q}}$. One can rewrite $\operatorname{Bs}\left|\mathcal{I}_{x}^{t+} L\right|_{\mathbb{Q}}$ as the intersection of the base locus $\operatorname{Bs}\left|\mathcal{I}_{x}^{p}(q L)\right|$, after all integers $p, q \geq 1$ such that $p>q t$. Since

$$
\oplus_{n \geq 0}\left\{s \in R_{n} ; \operatorname{ord}_{x}(s)>n t\right\}
$$

is a graded ring and $X$ is Noetherian, there exists $r \geq 1$ such that $\operatorname{Bs}\left|\mathcal{I}_{x}^{t+} L\right|_{\mathbb{Q}}=\operatorname{Bs}\left|\mathcal{I}_{x}^{\lfloor r t\rfloor+1}(r L)\right|$.

Example 2.1. Suppose $L$ is semiample. Let $\phi: X \rightarrow Y$ be the Iitaka map of $L$. Then Bs $\left|\mathcal{I}_{x}^{0+} L\right|_{\mathbb{Q}}=$ $\phi^{-1} \phi(x)$. It is connected, since $\phi$ is a contraction.

We have $r L \sim f^{*} A$ for some $r \geq 1$ and $A$ ample on $Y$. Then for every $t \geq 0$, we have $\operatorname{Bs}\left|\mathcal{I}_{x}^{t+} L\right|_{\mathbb{Q}} \subseteq f^{-1}\left(\operatorname{Bs}\left|\mathcal{I}_{f(x)}^{r t+} A\right|_{\mathbb{Q}}\right)$, and equality holds if $f$ is smooth at $x$.

Lemma 2.2. Given $u \geq 0$, there exists $\epsilon(u)>0$ such that $\mathrm{Bs}\left|\mathcal{I}_{x}^{t+} L\right|_{\mathbb{Q}}$ is constant for $t \in[u, u+\epsilon(u))$. Proof. Denote $B_{t}=\operatorname{Bs}\left|\mathcal{I}_{x}^{t+} L\right|_{\mathbb{Q}}$. We have $B_{u}=\cap_{l \geq 1} B_{u+\frac{1}{l}}$. By monotonicity, and since $X$ is Noetherian, there exists $l \geq 1$ such that $B_{u}=B_{u+\frac{1}{l}}$.

Lemma 2.3. There exists a constant $c$, depending only on $X$ and $L$, such that $\operatorname{Bs}\left|\mathcal{I}_{x}^{t+} L\right|_{\mathbb{Q}}=X$ for every $x \in X$ and $t \geq c$.

Proof. By Chow's Lemma and Hironaka's resolution of singularities, there exists a proper birational morphism $\mu: X^{\prime} \rightarrow X$ such that $X^{\prime}$ is smooth and projective. Let $A^{\prime}$ be very ample on $X^{\prime}$. Let $n \geq 1$ and $0 \neq s \in \Gamma\left(X, \mathcal{O}_{X}(n L)\right)$. Choose a point $x^{\prime} \in \mu^{-1}(x)$. Then $0 \neq \mu^{*} s \in \Gamma\left(X^{\prime}, \mathcal{O}_{X^{\prime}}\left(n \mu^{*} L\right)\right)$ and

$$
\operatorname{ord}_{x}(s) \leq \operatorname{ord}_{x^{\prime}}\left(\mu^{*} s\right) \leq n\left(\mu^{*} L \cdot A^{\prime d-1}\right),
$$

where the last inequality follows from Lemma 1.1. Thus Bs $\left|\mathcal{I}_{x}^{t+} L\right|_{\mathbb{Q}}=X$ for $t \geq\left(\mu^{*} L \cdot A^{\prime d-1}\right)$.

Recall that the codimension at $x$ of a closed subset $x \in Y \subseteq X$ is the smallest codimension of an irreducible component of $Y$ passing through $x$. Since $X$ is irreducible of $\operatorname{dimension} d \operatorname{codim}_{x}(Y \subseteq$ $X)=d-\operatorname{dim}_{x}(Y)$. When the ambient space $X$ is fixed, we will drop it from notation.

Definition 2.4. For $i \geq 1$, the $i$-th successive minimum of $L$ at $x$ is defined by

$$
\epsilon_{i}(L, x)=\inf \left\{t \geq 0 ; \operatorname{codim}_{x} \operatorname{Bs}\left|\mathcal{I}_{x}^{t+} L\right|_{\mathbb{Q}}<i\right\} .
$$

The definition makes sense, since $\operatorname{codim}_{x} \operatorname{Bs}\left|\mathcal{I}_{x}^{t+} L\right|_{\mathbb{Q}}=0$ for $t \gg 0$. The infimum is a minimum, by Lemma 2.2. Note that $\operatorname{codim}_{x} \operatorname{Bs}\left|\mathcal{I}_{x}^{t+} L\right|_{\mathbb{Q}}$ is strictly less than $i$ for $t \geq \epsilon_{i}(L, x)$, and at least $i$ for $0 \leq t<\epsilon_{i}(L, x)$. We obtain a chain of real numbers

$$
\epsilon_{1}(L, x) \geq \cdots \geq \epsilon_{d}(L, x) \geq \epsilon_{d+1}(L, x)=0 .
$$

Note that $\operatorname{codim}_{x} \operatorname{Bs}\left|\mathcal{I}_{x}^{t+} L\right|_{\mathbb{Q}}=0$ for $t \geq \epsilon_{1}(L, x)$, and $\operatorname{codim}_{x} \operatorname{Bs}\left|\mathcal{I}_{x}^{t+} L\right|_{\mathbb{Q}}=i$ if and only if $\epsilon_{i}(L, x)>$ $t \geq \epsilon_{i+1}(L, x)$.

Remark 2.5. One may similarly define successive minima $\epsilon_{i}(R, x)$ in points $x \in X$, associated to a graded subalgebra $R \subseteq R(L)$. For example, when $R$ is the image of the restriction $R\left(X^{\prime}, L\right) \rightarrow$ $R\left(X,\left.L^{\prime}\right|_{X}\right)$, where $X \subset X^{\prime}$ is a closed embedding and $L^{\prime}$ is a Cartier divisor on $X^{\prime}$. The successive minima of such subalgebras appear in Lemma 4.1, for example. 
Lemma 2.6. Let $L, L^{\prime}$ be Cartier divisors on $X$, let $x \in X$ be a closed point and $1 \leq i \leq d$.

a) $\epsilon_{i}(q L, x)=q \epsilon_{i}(L, x)$ for every integer $q \geq 1$.

b) If $L \sim_{\mathbb{Q}} L^{\prime}$, then $\epsilon_{i}(L, x)=\epsilon_{i}\left(L^{\prime}, x\right)$.

c) Suppose $\epsilon_{i}(L, x)>0$ and $\epsilon_{i}\left(L^{\prime}, x\right)>0$. Then $\epsilon_{i}\left(L+L^{\prime}, x\right) \geq \epsilon_{i}(L, x)+\epsilon_{i}\left(L^{\prime}, x\right)$.

Proof. Property a) follows from Bs $\left|\mathcal{I}_{x}^{t+}(q L)\right|_{\mathbb{Q}}=\operatorname{Bs}\left|\mathcal{I}_{x}^{\frac{t}{q}+} L\right|_{\mathbb{Q}}$, and b) from Bs $\left|\mathcal{I}_{x}^{t+} L\right|_{\mathbb{Q}}=\operatorname{Bs}\left|\mathcal{I}_{x}^{t+} L^{\prime}\right|_{\mathbb{Q}}$ for all $t \geq 0$.

c) Let $0 \leq t<\epsilon_{i}(L, x)$ and $0 \leq t^{\prime}<\epsilon_{i}\left(L^{\prime}, x\right)$. Then both $\operatorname{Bs}\left|\mathcal{I}_{x}^{t+} L\right|_{\mathbb{Q}}$ and $\operatorname{Bs}\left|\mathcal{I}_{x}^{t^{\prime}+} L^{\prime}\right|_{\mathbb{Q}}$ have codimension at $x$ at least $i$. Since

$$
\operatorname{Bs}\left|\mathcal{I}_{x}^{\left(t+t^{\prime}\right)+}\left(L+L^{\prime}\right)\right|_{\mathbb{Q}} \subseteq \operatorname{Bs}\left|\mathcal{I}_{x}^{t+} L\right|_{\mathbb{Q}} \cup \operatorname{Bs}\left|\mathcal{I}_{x}^{t^{\prime}+} L^{\prime}\right|_{\mathbb{Q}}
$$

the codimension at $x$ of the left hand side is at least $i$. Therefore $t+t^{\prime}<\epsilon_{i}\left(L+L^{\prime}, x\right)$. Letting $t$ and $t^{\prime}$ approach $\epsilon_{i}(L, x)$ and $\epsilon_{i}\left(L^{\prime}, x\right)$, respectively, we obtain the claim.

In particular, we may define the $i$-th successive minimum of a $\mathbb{Q}$-Cartier divisor $L$ at a point $x \in X$ to be $\epsilon_{i}(L, x)=\frac{1}{q} \epsilon_{i}(q L, x)$, where $q$ is a positive integer such that $q L$ is Cartier.

Lemma 2.7. Let $f: X^{\prime} \rightarrow X$ be a proper contraction of algebraic varieties, which is smooth at a point $x^{\prime} \in X^{\prime}$. Let $L$ be a Cartier divisor on $X$. Then $\epsilon_{i}\left(L, f\left(x^{\prime}\right)\right)=\epsilon_{i}\left(f^{*} L, x^{\prime}\right)$ for all $i$.

Proof. Denote $L^{\prime}=f^{*} L$ and $x=f\left(x^{\prime}\right)$. Since $f$ is a contraction, $f^{*}$ induces isomorphisms $\Gamma(n L) \stackrel{\sim}{\rightarrow} \Gamma\left(n L^{\prime}\right)(n \geq 1)$. Since $f^{*}$ maps $\mathcal{I}_{x}^{p}$ into $\mathcal{I}_{x^{\prime}}^{p}$, it induces injective homomorphisms

$$
\Gamma\left(\mathcal{I}_{x}^{p}(q L)\right) \rightarrow \Gamma\left(\mathcal{I}_{x^{\prime}}^{p}\left(q L^{\prime}\right)\right)(p, q \geq 1) .
$$

These are also surjective if $f$ is smooth at $x^{\prime}$, since in this case the order of a section $s$ at $x$ coincides with the order of $f^{*} s$ at $x^{\prime}$. We obtain isomorphisms

$$
f^{*}: \Gamma\left(\mathcal{I}_{x}^{p}(q L)\right) \stackrel{\sim}{\rightarrow} \Gamma\left(\mathcal{I}_{x^{\prime}}^{p}\left(q L^{\prime}\right)\right)(p, q \geq 1) .
$$

Therefore $\operatorname{Bs}\left|\mathcal{I}_{x^{\prime}}^{t+} L^{\prime}\right|_{\mathbb{Q}}=f^{-1}\left(\operatorname{Bs}\left|\mathcal{I}_{x}^{t+} L\right|_{\mathbb{Q}}\right)$. Since $f$ is smooth at $x^{\prime}$, we obtain

$$
\operatorname{codim}_{x^{\prime}} \operatorname{Bs}\left|\mathcal{I}_{x^{\prime}}^{t+} L^{\prime}\right|_{\mathbb{Q}}=\operatorname{codim}_{x} \operatorname{Bs}\left|\mathcal{I}_{x}^{t+} L\right|_{\mathbb{Q}} \text {. }
$$

Therefore $\epsilon_{i}(L, x)=\epsilon_{i}\left(f^{*} L, x^{\prime}\right)$ for all $i$.

Lemma 2.8. $\epsilon_{i}(L, x)>0$ if and only if there exist integers $p, q \geq 1$ such that $\operatorname{codim}_{x} \operatorname{Bs}_{\mathcal{I}_{x}^{p}}(q L) \mid \geq i$. Moreover, in this case we have

$$
\epsilon_{i}(L, x)=\sup \left\{\frac{p}{q} ; p, q \geq 1, \operatorname{codim}_{x} \operatorname{Bs}\left|\mathcal{I}_{x}^{p}(q L)\right| \geq i\right\} .
$$

Proof. Denote $\epsilon_{i}=\epsilon_{i}(L, x)$.

Step 1: $\operatorname{codim}_{x} \operatorname{Bs}\left|\mathcal{I}_{x}^{p}(q L)\right|<i$ for every $p, q \geq 1$ such that $p>q \epsilon_{i}$. Indeed, $\operatorname{Bs}\left|\mathcal{I}_{x}^{p}(q L)\right|$ contains $\operatorname{Bs}\left|\mathcal{I}_{x}^{\epsilon_{i}+} L\right|_{\mathbb{Q}}$, which has codimension at $x$ strictly less than $i$.

Step 2: Let $0 \leq t<\epsilon_{i}$. Then there exist $p, q \geq 1$ with $t<\frac{p}{q} \leq \epsilon_{i}(L, x)$ and $\operatorname{codim}_{x} \operatorname{Bs}\left|\mathcal{I}_{x}^{p}(q L)\right| \geq i$. Indeed, we have $\operatorname{codim}_{x} \operatorname{Bs}\left|\mathcal{I}_{x}^{t+} L\right|_{\mathbb{Q}} \geq i$. There exists $r \geq 1$ such that $\operatorname{Bs}\left|\mathcal{I}_{x}^{t+} L\right|_{\mathbb{Q}}=\operatorname{Bs}\left|\mathcal{I}_{x}^{\lfloor r t\rfloor+1}(r L)\right|$. Let $q=r$ and $p=\lfloor r t\rfloor+1$. By construction, $t<\frac{p}{q}$. By the first step, $\frac{p}{q} \leq \epsilon_{i}$.

If $\epsilon_{i}>0$, there exist $p, q \geq 1$ such that $\operatorname{codim}_{x} \operatorname{Bs}\left|\mathcal{I}_{x}^{p}(q L)\right| \geq i$, by Step 2. Conversely, the latter implies $\epsilon_{i} \geq \frac{p}{q}>0$, by Step 1. The two steps give that $\epsilon_{i}$ is the supremum after all such $\frac{p}{q}$.

Lemma 2.9. $\epsilon_{1}(L, x)=0$ if and only if one of the following holds:

a) $\kappa(L)=-\infty$, or

b) $\kappa(L)=0$ and every $0 \neq s \in \Gamma\left(X, \mathcal{O}_{X}(n L)\right)(n \geq 1)$ is invertible at $x$. 
Proof. Suppose a) or b) holds, that is $\Gamma\left(\mathcal{I}_{x}(n L)\right)=0$ for $n \geq 1$. Therefore Bs $\left|\mathcal{I}_{x}^{0+} L\right|_{\mathbb{Q}}=X$. We obtain $\epsilon_{1}(L, x)=0$.

Conversely, suppose $\epsilon_{1}(L, x)=0$. We may assume we are not in case a), that is $\kappa(L) \geq 0$. Let $n \geq 1$ such that $\Gamma(n L) \neq 0$. If $\operatorname{dim}_{k} \Gamma(n L) \geq 2$, then $\Gamma\left(\mathcal{I}_{x}(n L)\right) \neq 0$. This implies $\epsilon_{1}(L, x) \geq \frac{1}{n}$, a contradiction. Therefore $\operatorname{dim}_{k} \Gamma(n L)=1$. Since again $\Gamma\left(X, \mathcal{I}_{x}(n L)\right)=0$, we have $\Gamma(n L)=k s_{n}$ with $s_{n}(x) \neq 0$.

In particular, $\epsilon_{1}(L, x)$ is zero if $\kappa(L)<0$, and otherwise equals

$$
\sup \left\{\frac{\operatorname{ord}_{x}(s)}{n} ; 0 \neq s \in \Gamma(n L), n \geq 1\right\} .
$$

We call $\epsilon_{1}(L, x)$ the width of $L$ at the point $x$, also denoted by $\operatorname{width}_{x}(L)$. If $x \in X$ is a smooth point, then $\epsilon_{1}(L, x)$ coincides with the width of $L$ at the geometric valuation induced by the exceptional divisor of the blow-up of $X$ at $x$ (see [1, Section 2]).

By Lemma 2.8, $\epsilon_{d}(L, x)>0$ if and only if there exist integers $p, q \geq 1$ such that $\operatorname{Bs}\left|\mathcal{I}_{x}^{p}(q L)\right|=\{x\}$ near $x$, and in this case, $\epsilon_{d}(L, x)$ is the supremum of $\frac{p}{q}$ after all such $p, q$. We call $\epsilon_{d}(L, x)$ the Seshadri constant of $L$ at $x$, since we will see later (Proposition 2.20) that in the classical setting, it coincides with the Seshadri constant introduced by Demailly.

Lemma 2.10. $\epsilon_{d}(L, x)>0$ if and only if there exists $n \geq 1$ such that $x \notin \operatorname{Bs}|n L|$ and the induced rational map $\phi=\phi_{|n L|}: X \rightarrow X_{n} \subseteq|n L|$ satisfies $\phi^{-1} \phi(x)=\{x\}$ near $x$. In particular, $L$ is big.

Proof. Suppose $\epsilon_{d}(L, x)>0$. Then there exists $p, q \geq 1$ such that $\operatorname{Bs}\left|\mathcal{I}_{x}^{p}(q L)\right|=\{x\}$ near $x$. By Lemma 1.4, there exists $l \geq 1$ such that Bs $|q l L|$ is empty in a neighborhood of $x$. Denote $n=q l$. Let $\phi=\phi_{|n L|}: X \rightarrow X_{n} \subseteq|n L|$ be the induced rational map. It is regular on the open dense subset $U=X \backslash \operatorname{Bs}|n L|$, which contains $x$. Moreover, Bs $\left|\mathcal{I}_{x}(n L)\right| \cap U=\phi^{-1} \phi(x) \cap U$. Then

$$
0=\operatorname{dim}_{x} \operatorname{Bs}\left|\mathcal{I}_{x}(n L)\right|=\operatorname{dim}_{x} \phi^{-1} \phi(x) \geq \operatorname{dim} X-\operatorname{dim} X_{n} .
$$

Therefore $\phi^{-1} \phi(x)=\{x\}$ near $x$, and $\operatorname{dim} X_{n}=d$. In particular, $L$ is big.

Conversely, suppose $\phi=\phi_{|n L|}: X \rightarrow X_{n} \subseteq|n L|$ is regular near $x$ and $\phi^{-1} \phi(x)=\{x\}$ near $x$. Then $\operatorname{Bs}\left|\mathcal{I}_{x}(n L)\right|$ equals $\{x\}$ near $x$. Therefore $\epsilon_{d}(L, x) \geq \frac{1}{n}>0$.

The following lemma is a Successive minima version of [8, Proposition 6.4]

Lemma 2.11. Let $X$ be normal. Let $x \notin \operatorname{Bs}|L|_{\mathbb{Q}}$. For $n \geq 1$ such that $x \notin \operatorname{Bs}|n L|$, let $\phi_{|n L|}: X \rightarrow-\rightarrow$ $X_{n} \subseteq|n L|$ be the induced rational map. Let $\mu_{n}: Y_{n} \rightarrow X$ be the normalization of the graph of $\phi_{|n L|}$. In the mobile-fixed decomposition $\left|\mu_{n}^{*}(n L)\right|=\left|M_{n}\right|+F_{n}$, the mobile part is base point free. Moreover, $\mu_{n}$ is an isomorphism over a neighborhood of $x$. Then $\epsilon_{i}(L, x)$ is the supremum of $\frac{\epsilon_{i}\left(M_{n}, x\right)}{n}$, after all such $n$.

Proof. Since $x \notin F_{n} \in\left|n \mu_{n}^{*} L-M_{n}\right|$, we have Bs $\left|\mathcal{I}_{x}^{t+}\left(n \mu^{*} L\right)\right|_{\mathbb{Q}} \subseteq \operatorname{Bs}\left|\mathcal{I}_{x}^{t+} M_{n}\right|_{\mathbb{Q}}$ near $x$. Therefore $\epsilon_{i}\left(M_{n}, x\right) \leq \epsilon_{i}\left(n \mu^{*} L, x\right)=n \epsilon_{i}(L, x)$.

Suppose now $t<\epsilon_{i}(L, x)$. There exist integers $p, q \geq 1$ with $p>q t$ and $\operatorname{Bs}\left|\mathcal{I}_{x}^{t+} L\right|_{\mathbb{Q}}=\operatorname{Bs}\left|\mathcal{I}_{x}^{p}(q L)\right|$. The identifications $\Gamma\left(Y_{q}, \mathcal{O}_{Y_{q}}\left(M_{q}\right)\right)=\Gamma\left(Y_{q}, \mathcal{O}_{Y_{q}}\left(\mu_{q}^{*} q L\right)\right)=\Gamma\left(X, \mathcal{O}_{X}(q L)\right)$ induce an identification $\Gamma\left(Y_{q}, \mathcal{I}_{x \in Y_{q}}^{p}\left(M_{q}\right)\right)=\Gamma\left(X, \mathcal{I}_{x}^{p}(q L)\right)$. Therefore $\operatorname{codim}_{x} \operatorname{Bs}\left|\mathcal{I}_{x}^{p}\left(M_{q}\right)\right| \geq i$. We obtain $p \leq \epsilon_{i}\left(M_{q}, x\right)$, that is $t<\frac{p}{q} \leq \frac{\epsilon_{i}\left(M_{q}, x\right)}{q}$.

The supremum is in fact a limit.

Successive minima at a very general point. Contrary to the usual Seshadri constant, $\epsilon_{i}(L, x)$ is not lower semicontinuous with respect to $x \in X$ in general. For example, $\epsilon_{1}(L, x)$ can be upper semicontinuous. But one can show a weaker property, that is $\epsilon_{i}(L, x)$ is independent of the choice of a very general point $x$. 
Definition-Proposition 2.12. There exists a countable intersection of open dense subsets $V \subseteq X$ such that the correspondence $V \ni x \mapsto \epsilon_{i}(L, x)$ is constant. The common value does not depend on $V$, and is denoted by $\epsilon_{i}(L)$.

Proof. Let $p_{1}, p_{2}: X \times X \rightarrow X$ be the natural projections, let $\delta: X \rightarrow X \times X$ be the diagonal embedding, let $\Delta \subset X \times X$ be the diagonal. Let $p, q \geq 1$ be integers. Let $B^{p q}$ be the locus where the composition $p_{2}^{*} p_{2 *} \mathcal{I}_{\Delta}^{p}\left(q p_{1}^{*} L\right) \rightarrow \mathcal{I}_{\Delta}^{p}\left(q p_{1}^{*} L\right) \subset \mathcal{O}\left(q p_{1}^{*} L\right)$ is not surjective. By the definition, $B^{p q}$ contains $\Delta$. There exists an open dense subset $U^{p q} \subseteq X$ such that the following properties hold:

1) $p_{2 *}\left(\mathcal{I}_{\Delta}^{p}\left(q p_{1}^{*} L\right)\right) \otimes k(x) \stackrel{\sim}{\rightarrow} \Gamma\left(\mathcal{I}_{x}^{p}(q L)\right)$ for every $x \in U^{p q}$.

2) If $Y$ is an irreducible component of $B^{p q}$ which contains $\Delta$, then $p_{2}: Y \rightarrow X$ is flat over $U^{p q}$. In particular, $Y \cap p_{2}^{-1}(x)$ is equi-dimensional, of dimension $\operatorname{dim} Y-d$, for every $x \in U^{p q}$.

3) $\delta\left(U^{p q}\right)$ intersects only the irreducible components of $B^{p q}$ which contain $\Delta$.

Indeed, conditions 1) and 2) are open dense in $X$. For 3), we remove from $X$ the $\delta$-preimage of the trace on $\Delta$ of the irreducible components of $B^{p q}$ which do not contain $\Delta$. By 1), the natural inclusion $\operatorname{Bs}\left|\mathcal{I}_{x}^{p}(q L)\right| \subseteq B^{p q} \cap p_{2}^{-1}(x)$ is an equality for every $x \in U^{p q}$. By 2) and 3), we have

$$
\operatorname{dim}_{\delta(x)} B^{p q} \cap p_{2}^{-1}(x)=\operatorname{codim}\left(\Delta \subseteq B^{p q}\right) \forall x \in U^{p q} .
$$

Let $V=\cap_{p, q \geq 1} U^{p q}$. It is dense in $X$, a countable intersection of open subsets of $X$. We claim that $\epsilon_{i}(L, \cdot)$ is constant for $x \in V$. Suffices to show that if $x \in V, t \geq 0$ and $\epsilon_{i}(L, x)>t$, then $\epsilon_{i}(L, y)>t$ for every $y \in V$. Indeed, $\epsilon_{i}(L, x)>t$ implies that there exists $\frac{p}{q}>t$ such that $\operatorname{codim}_{x} \operatorname{Bs}_{\mathcal{I}_{x}^{p}}(q L) \mid \geq i$. That is $\operatorname{codim}_{\delta(x)} B^{p q} \cap p_{2}^{-1}(x) \geq i$. From above, this is equivalent to $\operatorname{codim}\left(\Delta \subseteq B^{p q}\right) \geq i$. Arguing backwards, we see that $\operatorname{codim}_{y} \operatorname{Bs}\left|\mathcal{I}_{y}^{p}(q L)\right| \geq i$ for every $y \in V$. Therefore $\epsilon_{i}(L, y) \geq \frac{p}{q}>t$ for every $y \in V$.

Lemma 2.13. Suppose $|n L| \neq \emptyset$ and $\operatorname{dim} \phi_{|n L|}(X) \geq i$. Then $\epsilon_{i}(L) \geq \frac{1}{n}$.

Proof. Let $U_{n} \subseteq X$ be the open dense subset on which $\phi=\phi_{|n L|}: X \rightarrow X_{n} \subseteq|n L|$ is regular. We have Bs $\left|\mathcal{I}_{x}(n L)\right| \cap U_{n}=\phi^{-1} \phi(x) \cap U_{n}$ for every $x \in U_{n}$. We have $\operatorname{dim}_{x} \phi^{-1} \phi(x) \geq \operatorname{dim} X-\operatorname{dim} X_{n}$ for all $x \in U_{n}$, and equality holds for an open dense subset $U_{n}^{\prime} \subseteq U_{n}$. Let $x \in U_{n}^{\prime}$. Then

$$
\operatorname{codim}_{x} \operatorname{Bs}\left|\mathcal{I}_{x}(n L)\right|=\operatorname{codim}_{x} \phi^{-1} \phi(x)=\operatorname{dim} X_{n} \geq i \text {. }
$$

By Lemma 2.8, $\epsilon_{i}(L, x) \geq \frac{1}{n}$.

Lemma 2.14. $\epsilon_{i}(L)>0$ if and only if $\kappa(L) \geq i$.

Proof. Suppose $\kappa(L) \geq i$. There exists $n \geq 1$ such that $|n L| \neq \emptyset$, and if $X \rightarrow X_{n} \subseteq|n L|$ is the induced rational map, then $\operatorname{dim} X_{n}=\kappa(L) \geq i$. By Lemma 2.13, $\epsilon_{i}(L) \geq \frac{1}{n}$.

Suppose $\kappa(L)<i$. Suppose $\Gamma(n L) \neq 0$, let $\phi_{n}: X \rightarrow X_{n} \subseteq|n L|$ be the induced rational map. For general $x \in X$ we have

$$
\operatorname{codim}_{x} \operatorname{Bs}\left|\mathcal{I}_{x}(n L)\right|=\operatorname{codim}_{x} \phi_{n}^{-1} \phi_{n}(x)=\operatorname{dim} X_{n}<i .
$$

Therefore $\epsilon_{i}(L, x)<\frac{1}{n}$. Letting $n$ approach infinity, we obtain $\epsilon_{i}(L)=0$.

Let $\kappa(L)=\kappa$. If $\kappa=0$, then $\epsilon_{i}(L)=0$ for all $i$. If $\kappa \geq 1$, we obtain

$$
\epsilon_{1}(L) \geq \cdots \geq \epsilon_{\kappa}(L)>0=\epsilon_{\kappa+1}(L) .
$$

Lemma 2.15. Suppose $\kappa\left(L^{\prime}-L\right) \geq 0$. Then $\epsilon_{i}(L) \leq \epsilon_{i}\left(L^{\prime}\right)$ for all $i$.

Proof. We may suppose $\left|L^{\prime}-L\right|$ is not empty. Choose $C \in\left|L^{\prime}-L\right|$. Then $\operatorname{Bs}\left|\mathcal{I}_{x}^{p}\left(q L^{\prime}\right)\right| \subseteq \operatorname{Bs}\left|\mathcal{I}_{x}^{p}(q L)\right| \cup$ $\operatorname{Supp} C$. Therefore $\operatorname{Bs}\left|\mathcal{I}_{x}^{t+} L^{\prime}\right|_{\mathbb{Q}} \subseteq \operatorname{Bs}\left|\mathcal{I}_{x}^{t+} L\right|_{\mathbb{Q}} \cup \operatorname{Supp} C$. For $x \in X \backslash C$, we obtain $\operatorname{codim}_{x} \operatorname{Bs}\left|\mathcal{I}_{x}^{t+} L^{\prime}\right|_{\mathbb{Q}} \geq \operatorname{codim}_{x} \operatorname{Bs}\left|\mathcal{I}_{x}^{t+} L\right|_{\mathbb{Q}}$.

By definition, $\epsilon_{i}(L, x) \leq \epsilon_{i}\left(L^{\prime}, x\right)$ for all $i$. 
Lemma 2.16. Let $\epsilon_{i}(L)>0, \kappa\left(L^{\prime}\right) \geq 0$, and $\left|l L-l^{\prime} L^{\prime}\right| \neq \emptyset$ for some integers $l, l^{\prime} \geq 1$. Then

$$
\lim _{n \rightarrow \infty} \frac{\epsilon_{i}\left(n L+L^{\prime}\right)}{n}=\epsilon_{i}(L) .
$$

Proof. Since $\kappa\left(L^{\prime}\right) \geq 0$, we have $\epsilon_{i}\left(n L+L^{\prime}\right) \geq n \epsilon_{i}(L)$. Let $C \in\left|l L-l^{\prime} L^{\prime}\right|$. For $n \geq 1$, we have

$$
\left(l^{\prime} n+l\right) L \sim l^{\prime}\left(n L+L^{\prime}\right)+C .
$$

Therefore $\left(l^{\prime} n+l\right) \epsilon_{i}(L) \geq l^{\prime} \epsilon_{i}\left(n L+L^{\prime}\right)$ by Lemma 2.15. We obtain

$$
0 \leq \epsilon_{i}\left(n L+L^{\prime}\right)-n \epsilon_{i}(L) \leq \frac{l \epsilon_{i}(L)}{l^{\prime}} .
$$

Dividing by $n$ and letting $n$ approach $+\infty$, we obtain the claim.

Proposition 2.17. Suppose $X$ is projective. Let $L \equiv L^{\prime}$ be numerically equivalent big Cartier divisors on $X$. Then $\epsilon_{i}(L)=\epsilon_{i}\left(L^{\prime}\right)$ for every $i$.

Proof. Let $A$ be an ample divisor on $X$. Since $(n L+A)-n L^{\prime}$ is ample, $\epsilon_{i}(n L+A) \geq n \epsilon_{i}\left(L^{\prime}\right)$. By Lemma 2.16, we obtain $\epsilon_{i}(L) \geq \epsilon_{i}\left(L^{\prime}\right)$. The other inequality holds by the same argument.

In particular, if $X$ is projective and $L$ is big, $\epsilon_{1}(L)$ coincides with the invariant $m(L)$ introduced by Nakamaye [17, page 2].

We may rephrase [7, Proposition 2.3] and [18, Lemma 1.3] as follows:

Lemma 2.18. Let $X$ be a proper algebraic variety, let $L$ be a Cartier divisor, let $t \geq 0$. Let $x \in X$ be a very general point and $Z \subseteq \mathrm{Bs}\left|\mathcal{I}_{x}^{t+} L\right|_{\mathbb{Q}}$ an irreducible component containing $x$. Then for integers $p, q \geq 1$ and $s \in \Gamma\left(\mathcal{I}_{x}^{p}(q L)\right)$, we have $\operatorname{ord}_{Z}(s) \geq p-q t$.

Proof. Let $p_{1}, p_{2}: X \times X \rightarrow X$ be the natural projections, let $\delta: X \rightarrow X \times X$ be the diagonal embedding, let $\Delta \subset X \times X$ be the diagonal.

Let $p, q \geq 1$ be integers with $p>q t$. Let $B^{p q}$ be the locus where the composition $p_{2}^{*} p_{2 *} \mathcal{I}_{\Delta}^{p}\left(q p_{1}^{*} L\right) \rightarrow$ $\mathcal{I}_{\Delta}^{p}\left(q p_{1}^{*} L\right) \subset \mathcal{O}\left(q p_{1}^{*} L\right)$ is not surjective. There exists an open dense subset $X^{p q} \subseteq X$ such that

$$
p_{2 *}\left(\mathcal{I}_{\Delta}^{p}\left(q p_{1}^{*} L\right)\right) \otimes k(x) \stackrel{\sim}{\rightarrow} \Gamma\left(X, \mathcal{I}_{x}^{p}(q L)\right) \forall x \in X^{p q} .
$$

We deduce that Bs $\left|\mathcal{I}_{x}^{p}(q L)\right| \subseteq B^{p q} \cap p_{2}^{-1}(x)$ for all $x \in X$, and equality holds for $x \in X^{p q}$.

Define $B=\cap_{p>q t} B^{p q}$, a closed subset of $X \times X$. Denote $X^{0}=\cap_{p>q t} X^{p q}$, a countable intersection of open dense subsets of $X$. Then $\operatorname{Bs}\left|\mathcal{I}_{x}^{t+} L\right|_{\mathbb{Q}} \subseteq B \cap p_{2}^{-1}(x)$ for all $x \in X$, and equality holds for $x \in X^{0}$.

We have $\Delta \subseteq B$, so at least one irreducible component of $B$ contains $\Delta$. Let $B^{\prime}$ be the union of irreducible components of $B$ which do not contain $\Delta$. Then $\delta^{-1}\left(\Delta \cap B^{\prime}\right)$ is a proper closed subset of $X$. Its complement $U \subseteq X$ is an open dense subset. For every $x \in U$, an irreducible component of $B$ which contains $\delta(x)$ must also contain $\Delta$. We may further shrink $U$, so that $U$ is smooth.

Let $x \in U \cap X^{0}$. Let $Z$ be an irreducible component of $\operatorname{Bs}\left|\mathcal{I}_{x}^{t+} L\right|_{\mathbb{Q}}$ which contains $x$, let $0 \neq$ $s \in \Gamma\left(\mathcal{I}_{x}^{p}(q L)\right)$. There exists an irreducible component $\tilde{Z}$ of $B$, which contains $\delta(x)$, such that $Z \subseteq \tilde{Z} \cap p_{2}^{-1}(x)$. Since $\tilde{Z}$ contains $\delta(x)$ and $x \in U, \tilde{Z}$ contains $\Delta$. Since $x \in X^{p q}$, there exists $\tilde{s} \in \Gamma\left(X \times T, \mathcal{I}_{\Delta}^{p}\left(q p_{1}^{*} L\right)\right)$ with $\left.\tilde{s}\right|_{p_{2}^{-1}(x)}=s$, where $T$ is a neighborhood of $x$ in $U$, which we may suppose affine and smooth. Let $m=\operatorname{ord}_{\tilde{Z}} \tilde{s}$. Since $\tilde{Z}$ contains $\Delta, p_{1}: \tilde{Z} \rightarrow X$ is dominant. Let $D^{m}$ be a general differential operator of order at most $m$ on $T$. Then $D^{m} \tilde{s} \in \Gamma\left(X \times T, \mathcal{I}_{\Delta}^{p-m}\left(q p_{1}^{*} L\right)\right)$ does not vanish along $\tilde{Z} \cap X \times T$, by the argument of [7, Proposition 2.3] (the argument goes through even if $X$ is singular, since $T$ is affine smooth and $X \times T$ is smooth at the general point of $\Delta \cap X \times T)$. Therefore $\tilde{Z} \cap X \times T$ is not contained $B^{p-m, q}$. Therefore $p-m \leq q t$.

We conclude $\operatorname{ord}_{Z}(s)=\operatorname{ord}_{Z}\left(\left.\tilde{s}\right|_{p_{2}^{-1}(x)}\right) \geq \operatorname{ord}_{Z}(\tilde{s}) \geq \operatorname{ord}_{\tilde{Z}}(\tilde{s})=m \geq p-q t$. 
Seshadri constant as $d$-th minimum. Recall our notation: $X$ is a proper algebraic variety of dimension $d, L$ is a Cartier divisor on $X$, and $x \in X$ is a closed point.

Proposition 2.19 (Seshadri criterion). The divisor $L$ is ample if and only if $\epsilon_{d}(L, x)>0$ for any $x \in X$.

Proof. Suppose $L$ is ample. There exists $n \geq 1$ such that $n L$ is very ample. That is, $\mathcal{I}_{x}(n L)$ is generated by global sections, for every point $x \in X$. Then $\operatorname{Bs}\left|\mathcal{I}_{x}(n L)\right|=\{x\}$, hence $\epsilon_{d}(L, x) \geq \frac{1}{n}$ for every $x$.

Conversely, suppose $\epsilon_{d}(L, x)>0$ for any $x \in X$. To show that $L$ is ample, by Nakai-Moishezon's criterion, suffices to show that for every irreducible subvariety $Y \subseteq X$, of dimension $r \geq 1$, we have $\left(\left.L\right|_{Y}\right)^{r}>0$. Note that $0<\epsilon_{d}(L, x) \leq \epsilon_{r}\left(\left.L\right|_{Y}, x\right)$ for every $x \in Y$. If $r<d=\operatorname{dim} X$, we conclude by induction that $\left.L\right|_{Y}$ is ample, hence $\left(\left.L\right|_{Y}\right)^{r}>0$. It remains to deal with the case $Y=X$. Since $\epsilon_{d}(L, x)$ is positive at a very general point, $L$ is big by Lemma 2.14. Since $L$ is also nef by induction, we deduce $\left(L^{d}\right)>0$.

Proposition 2.20. Suppose $X$ is projective and $L$ is nef. Then

$$
\epsilon_{d}(L, x)=\inf _{C \ni x} \frac{(L \cdot C)}{\operatorname{mult}_{x}(C)}=\sup \{0\} \cup\left\{\frac{p}{q} ; p, q \geq 1, \Gamma(q L) \rightarrow \mathcal{O}_{x} / \mathcal{I}_{x}^{p+1} \text { surjective }\right\} .
$$

Denote by $\epsilon^{s}(L, x)$ the infimum after all integral curves passing through $x$, and by $\epsilon^{j}(L, x)$ the supremum in the claim. First, we show the following lemma:

Lemma 2.21. For a Cartier divisor $L$ on a proper $X$,

a) $\epsilon_{d}(L, x) \geq \epsilon^{j}(L, x)$,

b) if $L$ is nef, $\epsilon^{s}(L, x) \geq \epsilon_{d}(L, x) \geq \epsilon^{j}(L, x)$.

Proof. a) Let $p, q \geq 1$ such that $\Gamma(q L) \rightarrow \mathcal{O}_{x} / \mathcal{I}_{x}^{p+1}$ is surjective. Equivalently, $\mathcal{I}_{x}^{i}(q L)$ is globally generated at $x$ for every $0 \leq i \leq p$. Then $\operatorname{Bs}\left|\mathcal{I}_{x}^{p}(q L)\right|=\{x\}$ near $x$. Therefore $\frac{p}{q} \leq \epsilon_{d}(L, x)$. Taking the supremum after all $\frac{p}{q}$, we obtain $\epsilon_{d}(L, x) \geq \epsilon^{j}(L, x)$.

b) Suppose $L$ is nef. If $\epsilon_{d}(L, x)=0, \epsilon^{s}(L, x) \geq \epsilon_{d}(L, x)$ is clear. Assume $\epsilon_{d}(L, x)>0$ and let $0<t<\epsilon_{d}(L, x)$. Then Bs $\left|\mathcal{I}_{x}^{t+} L\right|_{\mathbb{Q}}=\{x\}$ near $x$. Let $C \subseteq X$ be an integral curve passing through $x$. Since $C$ is not contained in $\operatorname{Bs}\left|\mathcal{I}_{x}^{t+} L\right|_{\mathbb{Q}}$, there exists $n \geq 1$ and $D \in|n L|$ such that $\operatorname{ord}_{x}(D)>n t$ and $C$ is not contained in the support of $D$. Then

$$
n(L \cdot C)=(D \cdot C) \geq \operatorname{ord}_{x}(D) \cdot \operatorname{mult}_{x}(C)>n t \operatorname{mult}_{x}(C) .
$$

Therefore $t \leq \epsilon^{s}(L, x)$. Taking the supremum after all such $t$, we obtain $\epsilon^{s}(L, x) \geq \epsilon_{d}(L, x)$.

To prove Proposition 2.20, it is enough to show $\epsilon^{s}(L, x) \leq \epsilon^{j}(L, x)$ by Lemma 2.21. In fact, $\epsilon^{s}(L, x)=\epsilon^{j}(L, x)$ is well known at least for ample $L$ and smooth $x \in X$ (see [13, Theorem 5.1.17]).

Proof of Proposition 2.20. Let $f: Y \rightarrow X$ be the blow-up of $X$ at $x$, with exceptional divisor $E$. Note that $f^{*} L-\epsilon^{s}(L, x) E$ is nef, and $\epsilon^{s}(L, x) \geq 0$ is maximal with this property.

Let $A$ be an ample divisor on $X$. The augmented base locus $\mathbf{B}_{+}(L)$ of $L$ is defined by

$$
\mathbf{B}_{+}(L)=\bigcap_{l \in \mathbb{N}} \operatorname{Bs}|l L-A|_{\mathbb{Q}}
$$

which does not depend on the choice of $A$. By [15], 4],

$$
\mathbf{B}_{+}(L)=\bigcup_{V} V
$$

holds, where the union runs over the subvarieties $V \subseteq X$ such that $\left.L\right|_{V}$ is not big. 
First, assume $x \in \mathbf{B}_{+}(L)$. Then there exists a subvariety $V \subseteq X$ containing $x$ such that $\left.L\right|_{V}$ is not big. Since $L$ is nef, we have $\left(L^{\operatorname{dim} V} . V\right)=0$. Hence $\epsilon^{s}(L, x)=0$ follows from

$0 \leq\left(\left(f^{*} L-\epsilon^{s}(L, x) E\right)^{\operatorname{dim} V^{\prime}} \cdot V^{\prime}\right)=\left(L^{\operatorname{dim} V} \cdot V\right)-\epsilon^{s}(L, x)^{\operatorname{dim} V} \cdot \operatorname{mult}_{x}(V)=-\epsilon^{s}(L, x)^{\operatorname{dim} V} \cdot \operatorname{mult}_{x}(V) \leq 0$, where $V^{\prime} \subseteq Y$ is the strict transform of $V$. By Lemma $2.21 \mathrm{~b}$ ), all three invariants equal to 0 .

So we may assume $x \notin \mathbf{B}_{+}(L)$. If we replace $A$ with its suitable multiple, we can take $l \geq 1$ and an effective Cartier divisor $D$ such that $l L \sim A+D$ and $x \notin \operatorname{Supp} D$. Since $A$ is ample and $-E$ is $f$-ample, there exists an integer $a \geq 1$ such that $a f^{*} A-E$ is ample.

By Lemma 2.21 b), it suffices to show $\epsilon^{s}(L, x) \leq \epsilon^{j}(L, x)$, which follows from the following claim: if $p, q \geq 1$ are integers such that $p-1 \leq(q-l a) \epsilon^{s}(L, x)$, then $\Gamma(n q L) \rightarrow \mathcal{O}_{x} / \mathcal{I}_{x}^{n p+1}$ is surjective for $n \gg 0$. Indeed, the divisor

$$
f^{*}(q L-a D)-p E=\left(a f^{*} A-E\right)+\left((q-l a) f^{*} L-(p-1) E\right)
$$

is ample. By Serre vanishing, $H^{1}\left(Y, \mathcal{I}_{E}\left(f^{*}(n q L-n a D)-n p E\right)\right)=0$ for $n \gg 0$. We obtain an exact sequence

$$
\Gamma\left(Y, f^{*}(n q L-n a D)\right) \rightarrow \Gamma\left((n p+1) E, \mathcal{O}_{(n p+1) E}\right) \rightarrow H^{1}\left(Y, n q f^{*} L-n a D-(n p+1) E\right)=0 .
$$

Since $n \gg 0$, the hypothesis of Lemma 1.6 are satisfied. Therefore $\Gamma(n q L-n a D) \rightarrow \mathcal{O}_{x} / \mathcal{I}_{x}^{n p+1}$ is surjective for $n \gg 0$. Since $D$ is effective and away from $x$, we deduce that $\Gamma(n q L) \rightarrow \mathcal{O}_{x} / \mathcal{I}_{x}^{n p+1}$ is surjective. Therefore $\frac{p}{q} \leq \epsilon^{j}(L, x)$. Since $\frac{p}{q}$ can be arbitrary close to $\epsilon^{s}(L, x)$, we obtain $\epsilon^{s}(L, x) \leq$ $\epsilon^{j}(L, x)$.

The equalities in Proposition 2.20 hold on proper $X$ if $L$ is semiample:

Lemma 2.22. Suppose $X$ is proper and $L$ is semiample. Then

$$
\epsilon_{d}(L, x)=\inf _{C \ni x} \frac{(L \cdot C)}{\operatorname{mult}_{x}(C)}=\sup \{0\} \cup\left\{\frac{p}{q} ; p, q \geq 1, \Gamma(q L) \rightarrow \mathcal{O}_{x} / \mathcal{I}_{x}^{p+1} \text { surjective }\right\} .
$$

Proof. There exists a proper contraction $f: X \rightarrow Y$, with $Y$ projective, such that $n L \sim f^{*} A$ for some integer $n \geq 1$ and some ample divisor $A$ on $Y$. Let $\operatorname{Exc}(f)$ be the exceptional locus of $f$, that is the locus of points $x \in X$ such that $\operatorname{dim}_{x} f^{-1} f(x)>0$.

If $x \in \operatorname{Exc}(f)$, we see that $\epsilon^{s}(L, x)=\epsilon_{d}(L, x)=\epsilon^{j}(L, x)=0$. Suppose $x \notin \operatorname{Exc}(f)$. Then $\operatorname{dim}_{x} f^{-1} f(x)=0$. By Lemma 1.2, $f$ is an isomorphism over a neighborhood of $f(x)$. It follows that $\epsilon_{d}(n L, x)=\epsilon_{d}(A, f(x))$ and $\epsilon^{*}(n L, x)=\epsilon^{*}(A, f(x))$ for $*=s, j$. We are reduced to the ample case, so we conclude from Proposition 2.20 .

Remark 2.23. By Lemma 2.22, the Seshadri constant of a semiample divisor $L$ at a point $x \in$ $X$ defined by Demailly [5, Theorem 6.4] coincides with $\epsilon_{d}(L, x)$. Combining with Lemma 2.11, we deduce that if $X$ is normal, projective, $L$ is big, and $x$ is a point away from the augmented base locus of $L$, then $\epsilon_{d}(L, x)$ coincides with the moving Seshadri constant $\epsilon_{m}(x, L)$ introduced by Nakamaye [16, Definition 0.4] (see also [8, Section 6]).

The interpretation of the Seshadri constant in terms of jets generation can be sharpened as follows: if $\epsilon_{d}(L, x)=\epsilon>0$, there exist constants $c_{1}(x) \geq 0$ and $c_{2}(x) \geq 0$ such that $\Gamma(q L) \rightarrow \mathcal{O}_{x} / \mathcal{I}_{x}^{p+1}$ is surjective for all integers $p, q \geq 1$ such that $p \geq c_{1}(x)$ and $q \epsilon-p \geq c_{2}(x)$. This statement reduces again to the ample case, which is the following Lemma.

Lemma 2.24. Suppose $L$ is ample. Let $\epsilon=\inf _{C \ni x} \frac{(L \cdot C)}{\operatorname{mult}_{x}(C)}$ the Seshadri constant of $L$ at $x$. Then there exist constants $c_{1}, c_{2} \geq 0$ such that for every integers $p, q \geq 1$ with $p \geq c_{1}$ and $q \epsilon-p \geq c_{2}$, the jet map $\Gamma(q L) \rightarrow \mathcal{O}_{X} / \mathcal{I}_{x}^{p+1}$ is surjective. 
Proof. Let $f: Y \rightarrow X$ be the blow-up of $X$ at $x$, with exceptional divisor $E$. Since $L$ is ample and $-E$ is $f$-ample, there exists an integer $a \geq 1$ such that $a f^{*} L-E$ is ample. By Nakai's ampleness criterion, $q f^{*} L-p E$ is ample if and only if $q \epsilon>p>0$. We have

$$
q f^{*} L-p E=m\left(a f^{*} L-E\right)+(q-m a) f^{*} L-(p-m) E .
$$

For $(q-m a) \epsilon \geq p-m \geq 0$, the divisor $(q-m a) f^{*} L-(p-m) E$ is nef. By Fujita's extension of Serre vanishing (see [13. Theorem 1.4.35]), there exists an integer $m=m\left(\mathcal{O}_{Y}, a f^{*} L-E\right)$ such that $H^{1}\left(q f^{*} L-p E\right)=0$ for $(q-m a) \epsilon \geq p-m \geq 0$.

We have $\mathcal{I}_{x}^{p+1}=f_{*}\left(\mathcal{I}_{E}^{p+1}\right)$ and $\bar{R}^{1} f_{*}\left(\mathcal{I}_{E}^{p+1}\right)=0$ for $p \geq c^{\prime}(x)$. Set $c_{1}=\max \left(c^{\prime}(x), m-1\right)$ and $c_{2}=m a \epsilon-m+1$.

Let $p, q \geq 1$ be integers with $p \geq c_{1}$ and $q \epsilon-p \geq c_{2}$. Then $p \geq c^{\prime}(x)$ and $(q-m a) \epsilon \geq p+1-m \geq 0$. The latter inequality implies that we have a surjection

$$
\Gamma\left(q f^{*} L\right) \rightarrow \Gamma\left(\mathcal{O}_{(p+1) E}\right) \rightarrow 0 .
$$

From the former inequality and Lemma 1.6, we deduce that $\Gamma(q L) \rightarrow \mathcal{O}_{X} / \mathcal{I}_{x}^{p+1}$ is surjective.

Successive minima in terms of jets generation. Once again, $X$ is a proper algebraic variety, $L$ is a Cartier divisor on $X$, and $x \in X$ is a closed point.

The following proposition is proved in [8, Proposition 6.6] for the moving Seshadri constant on smooth $X$ :

Proposition 2.25. We have $\epsilon_{d}(L, x)>0$ if and only if there exist $p, q \geq 1$ such that $\Gamma(q L) \rightarrow$ $\mathcal{O}_{x} / \mathcal{I}_{x}^{p+1}$ is surjective. Moreover, in this case we have

$$
\epsilon_{d}(L, x)=\sup \left\{\frac{p}{q} ; p, q \geq 1, \Gamma(q L) \rightarrow \mathcal{O}_{x} / \mathcal{I}_{x}^{p+1} \text { surjective }\right\}
$$

Proof. Denote by $\epsilon^{j}(L, x)$ the supremum in the claim. We obtain $\epsilon_{d}(L, x) \geq \epsilon^{j}(L, x)$ by Lemma 2.21 a). For the converse, we show the following claim:

Claim 2.26. If Bs $\left|\mathcal{I}_{x}^{r}(L)\right|=\{x\}$ near $x$ for some integer $r>0$, then $r \leq \epsilon^{j}(L, x)$.

Proof. We use the notation in Lemma 1.5. We identify $x$ with the point $g\left(f^{-1}(x)\right) \in Y$. Recall that we have a natural homomorphism $\Gamma\left(\mathcal{I}_{Z}^{q}(q L)\right) \rightarrow \Gamma(q A)$, which is an isomorphism for $q \geq c\left(\mathcal{I}_{Z}\right)$.

Since $\operatorname{Bs}\left|\mathcal{I}_{x}^{r}(L)\right|=\{x\}$ near $x$, Bs $\left|\mathcal{I}_{x}^{r}(A)\right|=\{x\}$ near $x \in Y$ and hence $r \leq \epsilon_{d}(A, x)$ holds. By applying Proposition 2.20 to the ample divisor $A, \epsilon_{d}(A, x)=\epsilon^{j}(A, x)$ holds. Hence, for any $0<t<r$, we can take $t<\frac{p}{q}<r$ such that $\Gamma(q A) \rightarrow \mathcal{O}_{x, Y} / \mathcal{I}_{x}^{p+1}$ is surjective. We note that we can take arbitrary large such $p, q$ by Lemma 2.24. So we take such $p, q$ with $q \geq c\left(\mathcal{I}_{Z}\right)$.

Since $q \geq c\left(\mathcal{I}_{Z}\right)$, we have an isomorphism $\Gamma\left(\mathcal{I}_{Z}^{q}(q L)\right) \stackrel{\sim}{\rightarrow} \Gamma(q A)$. Hence $\Gamma\left(\mathcal{I}_{Z}^{q}(q L)\right) \rightarrow \mathcal{O}_{x, X} / \mathcal{I}_{x}^{p+1}$ is surjective since so is $\Gamma(q A) \rightarrow \mathcal{O}_{x, Y} / \mathcal{I}_{x}^{p+1}$. Then $\Gamma(q L) \rightarrow \mathcal{O}_{x, X} / \mathcal{I}_{x}^{p+1}$ is also surjective since $x \notin Z$, and hence $t<\frac{p}{q} \leq \epsilon^{j}(L, x)$. Letting $t$ approach $r$, we obtain the claim.

For any $0 \leq t^{\prime}<\epsilon_{d}(L, x)$, we can take $t^{\prime}<\frac{r}{s}<\epsilon_{d}(L, x)$ such that $\operatorname{Bs}\left|\mathcal{I}_{x}^{r}(s L)\right|=\{x\}$ near $x$. Applying the above claim to $s L$, it holds that

$$
r \leq \epsilon^{j}(s L, x) \leq s \cdot \epsilon^{j}(L, x)
$$

where the last inequality follows from the definition of $\epsilon^{j}$. Hence $t^{\prime}<\frac{r}{s} \leq \epsilon^{j}(L, x)$ and the proposition follows by letting $t^{\prime}$ approach $\epsilon_{d}(L, x)$.

Lemma 2.27. Suppose $\epsilon_{1}(L, x)>0$. Then

$$
\epsilon_{1}(L, x)=\sup \left\{\frac{p}{q} ; p, q \geq 1, \Gamma\left(\mathcal{I}_{x}^{p}(q L)\right) \rightarrow \mathcal{I}_{x}^{p} / \mathcal{I}_{x}^{p+1} \text { non-zero }\right\} .
$$


Proof. Note that $\Gamma\left(\mathcal{I}_{x}^{p}(q L)\right) \rightarrow \mathcal{I}_{x}^{p} / \mathcal{I}_{x}^{p+1}$ is non-zero if and only if there exists $s \in \Gamma(q L)$ such that $\operatorname{ord}_{x}(s)=p$. In particular, $\epsilon_{1}(L, x) \geq \frac{p}{q}$. On the other hand, let $p, q \geq 1$ such that $\Gamma\left(\mathcal{I}_{x}^{p}(q L)\right) \neq 0$. Let $p^{\prime} \geq p$ be maximal such that $\Gamma\left(\mathcal{I}_{x}^{p^{\prime}}(q L)\right) \neq 0$. There exists $s \in \Gamma(q L)$ such that $\operatorname{ord}_{x}(s)=p^{\prime}$. Therefore $\frac{p}{q} \leq \frac{p^{\prime}}{q} \leq \epsilon_{1}(L, x)$. We conclude by Lemma 2.8.

Lemma 2.28. Suppose $\epsilon_{d}(L, x)>0$. Then

$$
\epsilon_{d}(L, x)=\sup \left\{\frac{p}{q} ; p, q \geq 1, \Gamma\left(\mathcal{I}_{x}^{p}(q L)\right) \rightarrow \mathcal{I}_{x}^{p} / \mathcal{I}_{x}^{p+1} \text { surjective }\right\}
$$

Proof. The inequality $\leq$ follows from Proposition 2.25. For the opposite inequality, suppose that $\Gamma\left(\mathcal{I}_{x}^{p}(q L)\right) \rightarrow \mathcal{I}_{x}^{p} / \mathcal{I}_{x}^{p+1}$ is surjective. By Nakayama's Lemma, $\mathcal{I}_{x}^{p}(q L)$ is generated by global sections at $x$. Therefore $\operatorname{Bs}\left|\mathcal{I}_{x}^{p}(q L)\right|=\{x\}$ near $x$. Therefore $p / q \leq \epsilon_{d}(L, x)$.

\section{Volume Versus the SuCCESSive minima}

The following well known statement may be considered as the analogue of Minkowski's first main theorem:

Proposition 3.1. Let $X$ be proper of dimension d, let $L$ be a Cartier divisor on $X$, let $x \in X$ be a closed point. Then $\operatorname{vol}(L) \leq \operatorname{mult}_{x}(X) \cdot \epsilon_{1}(L, x)^{d}$.

Proof. Let $t>\epsilon_{1}(L, x)$ be a real number. Let $n \geq 1$. We have $\Gamma\left(\mathcal{I}_{x}^{\lfloor n t\rfloor+1}(n L)\right)=0$, that is the jet map

$$
\Gamma(n L) \rightarrow \mathcal{O}_{x} / \mathcal{I}_{x}^{\lfloor n t\rfloor+1}
$$

is injective. Therefore $h^{0}(n L) \leq \operatorname{dim}_{k}\left(\mathcal{O}_{x} / \mathcal{I}_{x}^{\lfloor n t\rfloor+1}\right)$. For $n \gg 0$, the right hand side equals $P(\lfloor n t\rfloor)$, where $P(T)$ is a polynomial with leading term $\frac{e}{d !} T^{d}$, where $e=\operatorname{mult}_{x}(X)$. Therefore

$$
\limsup _{n \rightarrow \infty} \frac{h^{0}(n L)}{n^{d} / d !} \leq e \cdot t^{d} .
$$

Letting $t$ approach $\epsilon_{1}(L, x)$, we obtain the claim.

Lemma 3.2. Let $X$ be a proper variety of dimension $d$, let $\epsilon_{i}=\epsilon_{i}(L, x)$ be the Seshadri successive minima of a Cartier divisor $L$ at a closed point $x$. Then

$$
\operatorname{mult}_{x}(X) \cdot \prod_{i=1}^{d} \epsilon_{i}(L, x) \leq \operatorname{vol}(L) .
$$

In particular, $\operatorname{mult}_{x}(X) \cdot \epsilon_{d}(L, x)^{d} \leq \operatorname{vol}(L)$.

Proof. Suffices to show that for any real numbers $0 \leq t_{i}<\epsilon_{i}$, we have

$$
\operatorname{mult}_{x}(X) \cdot \prod_{i=1}^{d} t_{i}<\operatorname{vol}(L) .
$$

To prove this inequality, note first that there exist $D_{1}, \ldots, D_{d} \in|L|_{\mathbb{Q}}$ such that $\operatorname{ord}_{x}\left(D_{i}\right)>t_{i}$ and $\cap_{i=1}^{d} D_{i}=\{x\}$ near $x$. Indeed, there exists $D_{1} \in|L|_{\mathbb{Q}}$ with $\operatorname{ord}_{x}\left(D_{1}\right)>t_{1}$. Since $\operatorname{codim}_{x} \operatorname{Bs}\left|\mathcal{I}_{x}^{t_{2}+} L\right|_{\mathbb{Q}} \geq$ 2 , no irreducible component of $D_{1}$ through $x$ is contained in $\operatorname{Bs}\left|\mathcal{I}_{x}^{t_{2}+} L\right|_{\mathbb{Q}}$. There exists $D_{2} \in|L|_{\mathbb{Q}}$ with $\operatorname{ord}_{x}\left(D_{2}\right)>t_{2}$ and $\operatorname{codim}_{x}\left(D_{1} \cap D_{2}\right)=2$. Since $\operatorname{codim}_{x} \operatorname{Bs}\left|\mathcal{I}_{x}^{t_{3}+} L\right|_{\mathbb{Q}} \geq 3$, no irreducible component of $D_{1} \cap D_{2}$ through $x$ is contained in $\operatorname{Bs}\left|\mathcal{I}_{x}^{t_{3}+} L\right|_{\mathbb{Q}}$. Therefore there exists $D_{3} \in|L|_{\mathbb{Q}}$ with $\operatorname{ord}_{x}\left(D_{3}\right)>t_{3}$ and $\operatorname{codim}_{x}\left(D_{1} \cap D_{2} \cap D_{3}\right)=3$. Iterating the construction, we obtain the chain of divisors in $d$ steps.

There exists an integer $q \geq 1$ such that $q D_{i}$ are the zero divisors of some non-zero global sections $s_{i} \in \Gamma\left(X, \mathcal{I}_{x}^{\left\lfloor q t_{i}\right\rfloor+1}(q L)\right)$. Let $Z$ be the (possibly empty) subscheme $\cap_{i=1}^{d} q D_{i} \backslash\{x\} \subset X$. Let $f: Y \rightarrow$ 
$X$ be the blow-up of $X$ along $\mathcal{I}_{Z}$, let $E$ be the exceptional divisor on $Y$. Then $f^{*} s_{1}, \ldots, f^{*} s_{d}$ become global sections of $\mathcal{O}_{Y}\left(q f^{*} L-E\right)$, having no common zeroes near $E$. Therefore Bs $\left|q f^{*} L-E\right| \subseteq\{x\}$ (we identify $x$ with a point of $Y$ ). By Lemma 1.4, $M=q f^{*} L-E$ is semiample.

Set $D_{i}^{\prime}=f^{*} q D_{i}-E \in|M|$. By construction, $\cap_{i=1}^{d} D_{i}^{\prime}=\{x\} \subset Y$ as sets. Hence we have

$$
\left(M^{d}\right)=\left(D_{1}^{\prime} \cdot D_{2}^{\prime} \cdots D_{d}^{\prime}\right)=i\left(x, D_{1}^{\prime} \cdot D_{2}^{\prime} \cdots D_{d}^{\prime} ; Y\right) \geq \operatorname{mult}_{x}(Y) \cdot \prod_{i=1}^{d}\left(\left\lfloor q t_{i}\right\rfloor+1\right)>\operatorname{mult}_{x}(X) \cdot \prod_{i=1}^{d} q t_{i}
$$

by [10, page 233], where $i\left(x, D_{1}^{\prime} \cdot D_{2}^{\prime} \cdots D_{d}^{\prime} ; Y\right)$ is the intersection multiplicity. For $l \geq c\left(\mathcal{I}_{Z}\right)$, the natural homomorphism $\mathcal{I}_{Z}^{l} \rightarrow f_{*} \mathcal{O}_{Y}(-l E)$ is an isomorphism, hence so is

$$
\Gamma\left(X, \mathcal{I}_{Z}^{l}(l q L)\right) \stackrel{\sim}{\rightarrow} \Gamma\left(Y, \mathcal{O}_{Y}(l M)\right) .
$$

Therefore $q^{d} \operatorname{vol}(L)=\operatorname{vol}(q L) \geq \operatorname{vol}(M)=\left(M^{d}\right)>\operatorname{mult}_{x}(X) \cdot \prod_{i=1}^{d} q t_{i}$. Dividing out by $q$ and letting $t_{i}$ approach $\epsilon_{i}$, we obtain the claim.

Corollary 3.3. Let $X$ be an algebraic variety of dimension d, let $x$ be a closed point and $L$ a Cartier divisor on $X$. Then

$$
\epsilon_{d}(L, x) \leq \sqrt[d]{\frac{\operatorname{vol}(L)}{\operatorname{mult}_{x}(X)}} \leq \epsilon_{1}(L, x)
$$

\section{Minkowski's second main theorem for Seshadri successive minima.}

Lemma 3.4. Let $L$ be a Cartier divisor on a proper algebraic variety $X$. Let $\epsilon_{i}=\epsilon_{i}(L)$ be the Seshadri successive minima of $L$ at a very general point. Then

$$
h^{0}(L) \leq\left|\mathbb{Z}^{d} \cap \square\left(\epsilon_{1}, \ldots, \epsilon_{d}\right)\right| .
$$

Proof. Let $x \in X$ be a very general point.

Step 1: Let $p \geq 0$ be an integer. Then

$$
h^{0}\left(\mathcal{I}_{x}^{p}(L)\right)-h^{0}\left(\mathcal{I}_{x}^{p+1}(L)\right) \leq\left|\cap_{i=2}^{d}\left\{\alpha \in \mathbb{N}^{d}(p) ; \alpha_{i}+\cdots+\alpha_{d} \leq \epsilon_{i}\right\}\right| .
$$

Indeed, we have an exact sequence

$$
0 \rightarrow \Gamma\left(\mathcal{I}_{x}^{p+1}(L)\right) \rightarrow \Gamma\left(\mathcal{I}_{x}^{p}(L)\right) \stackrel{r}{\rightarrow} \mathcal{I}_{x}^{p} / \mathcal{I}_{x}^{p+1} \simeq \Gamma\left(\mathcal{O}_{\mathbb{P}^{d-1}}(p)\right)
$$

Therefore $h^{0}\left(\mathcal{I}_{x}^{p}(L)\right)-h^{0}\left(\mathcal{I}_{x}^{p+1}(L)\right)=\operatorname{dim}_{k} \operatorname{Im}(r)$. We may suppose $\operatorname{Im}(r) \neq 0$. In particular, $h^{0}\left(\mathcal{I}_{x}^{p}(L)\right)>0$, hence $p \leq \epsilon_{1}$. If $p \leq \epsilon_{d}$, the desired inequality becomes $\operatorname{dim} \operatorname{Im}(r) \leq\left|\mathbb{N}^{d}(p)\right|$, which follows from the inclusion $\operatorname{Im}(r) \subseteq \Gamma\left(\mathcal{O}_{\mathbb{P}^{d-1}}(p)\right)$. Therefore we may suppose $\epsilon_{d}<p \leq \epsilon_{1}$.

Denote by $J$ the set of indices $1<i \leq d$ such that $\epsilon_{i}<p$ and $\epsilon_{i}<\epsilon_{i-1}$. Fix $i \in J$. Since $\epsilon_{i}<\epsilon_{i-1}$, $\operatorname{Bs}\left|\mathcal{I}_{x}^{\epsilon_{i}+} L\right|_{\mathbb{Q}}$ has codimension $i-1$ at $x$. Choose an irreducible component $Z^{i-1}$, of codimension $i-1$, passing through $x$. On the blow-up of $X$ at $x$, the proper transform of $Z^{i-1}$ has in common with the exceptional locus $E \simeq \mathbb{P}^{d-1}$ at least one irreducible subvariety $W^{i-1} \subset \mathbb{P}^{d-1}$, of codimension $i-1$. Let $s \in \Gamma\left(\mathcal{I}_{x}^{p}(L)\right)$. By Lemma 2.18, $\operatorname{ord}_{Z^{i-1}}(s) \geq p-\epsilon_{i}$. Therefore the image of $r$ is contained in $\left\{P \in \Gamma\left(\mathcal{O}_{\mathbb{P}^{d-1}}(p)\right) ; \operatorname{ord}_{W^{i-1}}(P) \geq p-\epsilon_{i}\right\}$. We conclude

$$
\operatorname{Im}(r) \subseteq \cap_{i \in J}\left\{P \in \Gamma\left(\mathcal{O}_{\mathbb{P}^{d-1}}(p)\right) ; \operatorname{ord}_{W^{i-1}}(P) \geq p-\epsilon_{i}\right\}
$$

By Proposition 1.7, the right hand side has dimension at most

$$
\left|\cap_{i \in J}\left\{\alpha \in \mathbb{N}^{d}(p) ; \alpha_{i}+\cdots+\alpha_{d} \leq \epsilon_{i}\right\}\right| .
$$

From the definition of $J$, we see that

$$
\cap_{i \in J}\left\{\alpha \in \mathbb{N}^{d}(p) ; \alpha_{i}+\cdots+\alpha_{d} \leq \epsilon_{i}\right\}=\cap_{i=2}^{d}\left\{\alpha \in \mathbb{N}^{d}(p) ; \alpha_{i}+\cdots+\alpha_{d} \leq \epsilon_{i}\right\} .
$$

Therefore $\operatorname{dim}_{k} \operatorname{Im}(r) \leq\left|\cap_{i=2}^{d}\left\{\alpha \in \mathbb{N}^{d}(p) ; \alpha_{i}+\cdots+\alpha_{d} \leq \epsilon_{i}\right\}\right|$. 
Step 2: Let $p \geq 0$ be an integer. Then

$$
h^{0}(L)-h^{0}\left(\mathcal{I}_{x}^{p+1}(L)\right) \leq\left|\mathbb{Z}^{d} \cap \square\left(p, \epsilon_{2}, \ldots, \epsilon_{d}\right)\right| .
$$

Indeed, $h^{0}(L)-h^{0}\left(\mathcal{I}_{x}^{p+1}(L)\right)=\sum_{l=0}^{p} h^{0}\left(\mathcal{I}_{x}^{l}(L)\right)-h^{0}\left(\mathcal{I}_{x}^{l+1}(L)\right)$. Applying Step 1 to each term of the sum, we obtain the desired inequality.

Step 3) Set $p=\left\lfloor\epsilon_{1}\right\rfloor$ in Step 2. Then $p+1>\epsilon_{1}$, so $h^{0}\left(\mathcal{I}_{x}^{p+1}(L)\right)=0$. We obtain

$$
h^{0}(L) \leq\left|\mathbb{Z}^{d} \cap \square\left(\left\lfloor\epsilon_{1}\right\rfloor, \epsilon_{2}, \ldots, \epsilon_{d}\right)\right| .
$$

Since $\mathbb{Z}^{d} \cap \square\left(\left\lfloor t_{1}\right\rfloor, \ldots,\left\lfloor t_{d}\right\rfloor\right)=\mathbb{Z}^{d} \cap \square\left(t_{1}, \ldots, t_{d}\right)$, we obtain

$$
h^{0}(L) \leq\left|\mathbb{Z}^{d} \cap \square\left(\epsilon_{1}, \ldots, \epsilon_{d}\right)\right| .
$$

Proposition 3.5. Let $L$ be a Cartier divisor on a proper algebraic variety $X$, with Iitaka dimension $\kappa(L)=\kappa \geq 1$. Let $\epsilon_{i}=\epsilon_{i}(L)$ be the Seshadri successive minima of $L$ at a very general point. Then

$$
\limsup _{n \rightarrow \infty} \frac{h^{0}(n L)}{n^{\kappa} / \kappa !} \leq \kappa ! \cdot \operatorname{vol} \square\left(\epsilon_{1}, \ldots, \epsilon_{\kappa}\right) .
$$

In particular, if $L$ is big, $\operatorname{vol}(L) \leq d ! \cdot \operatorname{vol} \square\left(\epsilon_{1}, \ldots, \epsilon_{d}\right)$.

Proof. Suppose $t_{\kappa+1}=\cdots=t_{d}=0$. Then $\square\left(t_{1}, \ldots, t_{d}\right)=\square\left(t_{1}, \ldots, t_{\kappa}\right) \times 0$, where 0 is the origin in $\mathbb{R}^{d-\kappa}$. We have $\epsilon_{1} \geq \cdots \geq \epsilon_{\kappa}>0=\epsilon_{\kappa+1}=\cdots=\epsilon_{d}$. Since $\epsilon_{i}(n L)=n \epsilon_{i}(L)$, we obtain

$$
h^{0}(n L) \leq\left|\mathbb{Z}^{\kappa} \cap \square\left(n \epsilon_{1}, \ldots, n \epsilon_{\kappa}\right)\right| \forall n \geq 0
$$

As $n \rightarrow \infty$, the right hand side grows like $\operatorname{vol} \square\left(\epsilon_{1}, \ldots, \epsilon_{\kappa}\right) \cdot n^{\kappa}+O\left(n^{\kappa-1}\right)$. Therefore the claim holds.

Proposition 3.5 generalizes the following result of Nakamaye [17, Proof of Corollary 3]: if $L$ is an ample divisor on a smooth projective surface, then $\left(L^{2}\right) \leq 2 \epsilon_{1}(L) \epsilon_{2}(L)-\epsilon_{2}(L)^{2}$.

Combining Lemma 1.8, Propositions 3.2 and 3.5, we obtain the following equivalent of Minkowski's second theorem for successive minima:

Theorem 3.6. Let $L$ be a big Cartier divisor on a d-dimensional proper algebraic variety $X$. Then

$$
1 \leq \frac{\operatorname{vol}(L)}{\prod_{i=1}^{d} \epsilon_{i}(L)} \leq d !
$$

So the volume of $L$ is essentially the product of the successive Seshadri minima of $L$ at a very general point.

Remark 3.7. If $(X, L)=\left(\mathbb{P}^{d}, \mathcal{O}(1)\right), \epsilon_{i}(L)=1$ for any $i$. Hence $\frac{\operatorname{vol}(L)}{\prod_{i=1}^{d} \epsilon_{i}(L)}=1$. On the other hand, consider $(X, L)=\left(\left(\mathbb{P}^{1}\right)^{d}, \mathcal{O}\left(w_{1}, \ldots, w_{d}\right)\right)$, where $w_{1} \geq w_{2} \geq \cdots \geq w_{d}$ are positive integers. Then $\epsilon_{i}(L)=\sum_{j=i}^{d} w_{j}$ and $\operatorname{vol}(L)=d ! \prod_{i=1}^{d} w_{i}$ as we will see in Example 4.5. Hence

$$
\frac{\operatorname{vol}(L)}{\prod_{i=1}^{d} \epsilon_{i}(L)}=d ! \prod_{i=1}^{d} \frac{w_{i}}{\sum_{j=i}^{d} w_{j}}
$$

could be arbitrary close to $d$ ! if we take $w_{1} \gg w_{2} \gg \cdots \gg w_{d}$.

Thus the lower and upper bounds in Theorem 3.6 are sharp. We also note that the upper bound is not attained for $d \geq 2$ since vol $\square\left(\epsilon_{1}(L), \ldots, \epsilon_{d}(L)\right)<\prod_{i=1}^{d} \epsilon_{i}(L)$ by $\square\left(\epsilon_{1}(L), \ldots, \epsilon_{d}(L)\right) \subsetneq$ $\left[0, \epsilon_{1}(L)\right] \times \square\left(\epsilon_{2}(L), \ldots, \epsilon_{d}(L)\right)$. 


\section{SuCCESIVE MINIMA ON TORIC VARIETIES}

For standard terminology on toric varieties, the reader may consult [19]. Let $X=T_{N} \operatorname{emb}(\Delta)$ be a proper toric variety (normal), of dimension $d$. Let $L$ be a Cartier divisor on $X$. Modulo linear equivalence, we may suppose $L$ is torus invariant. Due to the torus action, $\epsilon_{i}(L, \cdot)$ is constant on $T_{N} \subset X$. Therefore $\epsilon_{i}(L)=\epsilon_{i}(L, 1)$, where 1 denotes the unit of the torus $T_{N}$. We will estimate $\epsilon_{i}(L, x)$ when either $x$ is a closed invariant point, or $x=1$.

At a closed invariant point. Let $x \in X$ be a closed invariant point. It corresponds to a top cone $\sigma \in \Delta($ top $)$, and we obtain an open affine neighborhood $x \in U_{\sigma}=\operatorname{Spec} k\left[M \cap \sigma^{\vee}\right]$. Denote by $S$ the semigroup $M \cap \sigma^{\vee} \backslash 0$, so that $I\left(x \in U_{\sigma}\right)=\oplus_{m \in S} k \cdot \chi^{m}$. For $p \geq 1$, denote $S^{(p)}=\left\{s_{1}+\cdots+s_{p} ; s_{1}, \ldots, s_{p} \in S\right\}$. Therefore $I\left(x \in U_{\sigma}\right)^{p}=\oplus_{m \in S^{(p)}} k \cdot \chi^{m}$. Since $L$ is Cartier, there exists $u \in M$ such that $\left(\chi^{u}\right)+\left.L\right|_{U_{\sigma}}=0$. We have $\square_{L}-u \subseteq \sigma^{\vee}$.

The subspace $\left\{s \in \Gamma\left(X, \mathcal{O}_{X}(q L)\right) ; \operatorname{ord}_{x}(s) \geq p\right\} \subseteq \Gamma\left(X, \mathcal{O}_{X}(q L)\right)$ is torus invariant. Therefore a basis over $k$ consists of monomials $\chi^{m}$ such that $m \in M \cap q \square_{L}$ and $m-q u \in S^{(p)}$.

Let $P=\operatorname{Conv}\left(S+\sigma^{\vee}\right)$ be the Newton polytope associated to $S \subset \sigma^{\vee}$. Let $B$ be the closure of the complement $\sigma^{\vee} \backslash P$. Then $B$ is compact and contains a relatively open neighborhood of the origin in $\sigma^{\vee}$. In particular, $\sigma^{\vee}=\cup_{t \geq 0} t B$. Note that $B$ may not be convex, but in case $x$ is a smooth point, $B$ is a unit simplex. Moreover, $d ! \cdot \operatorname{vol}_{M}(B)=\operatorname{mult}_{x}(X)$.

We claim that $S^{(p)} \subseteq M \cap p P \subseteq S^{(p-d+1)}$. Indeed, the first inclusion is clear. For the second, let $m \in M \cap p P$. Then $m=\sum_{i \in I} t_{i} m^{i}+m^{\prime}$, where $t_{i} \geq 0, m^{i} \in S, \sum_{i} t_{i}=p$ and $m^{\prime} \in \sigma^{\vee}$, and the cardinality of $I$ is at most $d$ (use Carathéodory's theorem). Then $m=\sum_{i}\left\lfloor t_{i}\right\rfloor m^{i}+\left(m^{\prime}+\sum_{i}\left\{t_{i}\right\} m^{i}\right)$, and $\sum_{i}\left\lfloor t_{i}\right\rfloor>\sum_{i}\left(t_{i}-1\right)=p-d$. Therefore $m \in S^{(p-d+1)}$.

We obtain $\Gamma\left(\mathcal{I}_{x}^{p}(q L)\right) \subseteq \oplus_{m \in M \cap q \square_{L} \cap p P+q u} k \cdot \chi^{m} \subseteq \Gamma\left(\mathcal{I}_{x}^{p-d+1}(q L)\right)$. Therefore the two algebras

$$
\oplus_{p>q t} \Gamma\left(\mathcal{I}_{x}^{p}(q L)\right) \subseteq \oplus_{p>q t} \oplus_{m \in M \cap q \square_{L} \cap p P+q u} k \cdot \chi^{m}
$$

have the same stable base locus. Denote $t^{+} P=\cap_{\epsilon>0}(t+\epsilon) P$. We deduce that near $x$, Bs $\left|\mathcal{I}_{x}^{t+} L\right|_{\mathbb{Q}}$ is the intersection of $\operatorname{Supp}\left(\chi^{m}\right)$, where $m \in\left(\square_{L}-u\right) \cap t^{+} P \cap M_{\mathbb{Q}}$ and $\left(\chi^{m}\right)$ is the effective $\mathbb{Q}$-divisor defined by $\chi^{m}$.

Lemma 4.1. Near $x, \operatorname{Bs}\left|\mathcal{I}_{x}^{t+} L\right|_{\mathbb{Q}}$ is the union of invariant closed irreducible subvarieties $x \in Y \subseteq X$ such that width $_{x}\left(\left.R(L)\right|_{Y}\right) \leq t$. If $L$ is ample, the latter inequality means width $_{x}\left(\left.L\right|_{Y}\right) \leq t$.

Proof. Bs $\left|\mathcal{I}_{x}^{t+} L\right|_{\mathbb{Q}}$ is the intersection of all $\operatorname{Supp}\left(\chi^{m}\right)$, where $\chi^{m} \in \Gamma\left(\mathcal{O}_{X}(q L)\right)$ with $\operatorname{ord}_{x}\left(\chi^{m}\right)>q t$. In particular, Bs $\left|\mathcal{I}_{x}^{t+} L\right|_{\mathbb{Q}}$ is torus invariant.

Let $x \in Y \subseteq X$ be a torus invariant subvariety. By definition, $\operatorname{width}_{x}\left(\left.R(L)\right|_{Y}\right)>t$ if and only if the subspace

$$
\left\{\left.s\right|_{Y} ; s \in \Gamma\left(X, \mathcal{O}_{X}(q L)\right),\left.s\right|_{Y} \neq 0, \operatorname{ord}_{x}\left(\left.s\right|_{Y}\right)>q t\right\} \subseteq \Gamma\left(Y, \mathcal{O}_{Y}\left(\left.q L\right|_{Y}\right)\right)
$$

contains a non-zero element for some $q$. This subspace is torus invariant, and hence has a basis consists of monomials. Thus this subspace has a non-zero element if and only if there exists $\chi^{m} \in$ $\Gamma\left(\mathcal{O}_{X}(q L)\right)$ with $\left.\chi^{m}\right|_{Y} \neq 0$ and $\operatorname{ord}_{x}\left(\left.\chi^{m}\right|_{Y}\right)>q t$. For monomials, the restriction to $Y$ does not change the order, that is, $\operatorname{ord}_{x}\left(\left.\chi^{m}\right|_{Y}\right)=\operatorname{ord}_{x}\left(\chi^{m}\right)$ if $\left.\chi^{m}\right|_{Y} \neq 0$. Hence $\operatorname{width}_{x}\left(\left.R(L)\right|_{Y}\right)>t$ if and only if there exists $\chi^{m} \in \Gamma\left(\mathcal{O}_{X}(q L)\right)$ with $\left.\chi^{m}\right|_{Y} \neq 0$ and $\operatorname{ord}_{x}\left(\chi^{m}\right)>q t$ for some $q$, which is equivalent to say that $Y$ is not contained in $\operatorname{Bs}\left|\mathcal{I}_{x}^{t+} L\right|_{\mathbb{Q}}$.

If $L$ is ample, the restriction map $\Gamma(q L) \rightarrow \Gamma\left(\left.q L\right|_{Y}\right)$ is surjective for any $q$, and therefore $\operatorname{width}_{x}\left(\left.R(L)\right|_{Y}\right)=\operatorname{width}_{x}\left(\left.L\right|_{Y}\right)$.

Lemma 4.2. Let $x \in Y \subseteq X$ be an invariant closed subvariety corresponding to a face $\tau \prec \sigma$. Then $\operatorname{width}_{x}\left(\left.R(L)\right|_{Y}\right)=\min \left\{t \geq 0 ;\left(\square_{L}-u\right) \cap \tau^{\perp} \subseteq t B \cap \tau^{\perp}\right\} \in \mathbb{Q}$. In particular, width $(L)=$ $\min \left\{t \geq 0 ; \square_{L}-u \subseteq t B\right\} \in \mathbb{Q}$. 
Proof. By Lemma 4.1, width ${ }_{x}\left(\left.R(L)\right|_{Y}\right) \leq t$ if and only if $Y \subseteq \operatorname{Bs}\left|\mathcal{I}_{x}^{t+} L\right|_{\mathbb{Q}}$, if and only if $\left(\square_{L}-u\right) \cap$ $t^{+} P \cap \tau^{\perp} \cap M_{\mathbb{Q}}=\emptyset$. Since $\square_{L}, u, P$ are rational, this condition is equivalent to $\left(\square_{L}-u\right) \cap t^{+} P \cap \tau^{\perp}=\emptyset$, which is equivalent to $\square_{L}-u \cap \tau^{\perp} \subseteq t B \cap \tau^{\perp}$.

We obtain the following proposition which generalizes [3, Corollary 4.2.2].

Proposition 4.3. $\epsilon_{i}(L, x)$ is the minimum of width $_{x}\left(\left.R(L)\right|_{Y}\right)$, after all closed irreducible invariant subvarieties $x \in Y \subseteq X$ of codimension $i-1$. In particular, $\epsilon_{i}(L, x) \in \mathbb{Q}$.

Suppose $L$ is ample. Then $\operatorname{width}_{x}\left(\left.R(L)\right|_{Y}\right)=\operatorname{width}_{x}\left(\left.L\right|_{Y}\right)$, and therefore $\epsilon_{i}(L, x)$ is the minimum of width $_{x}\left(\left.L\right|_{Y}\right)$, after all closed irreducible invariant subvarieties $x \in Y \subseteq X$ of codimension $i-1$. In particular, $\epsilon_{d}(L, x)=\min \{(L \cdot C) ; x \in C \subseteq X$ invariant curve $\}$. Moreover, we have inclusions

$$
\epsilon_{d} B \subseteq \square_{L}-u \subseteq \epsilon_{1} B
$$

and $\epsilon_{d}, \epsilon_{1}$ are maximal and minimal, respectively, with this property.

At a general point. Let $\square \subset M_{\mathbb{R}}$ be the moment polytope of $L$. Then $\square-\square \subset M_{\mathbb{R}}$ is a 0 -symmetric compact convex set, of dimension $\kappa=\kappa(L)$. The Minkowski successive minima of $(M, \square-\square)$ are

$$
\lambda_{i}=\lambda_{i}(M, \square-\square)=\sup \{t \geq 0 ; \operatorname{dim} M \cap t(\square-\square)<i\} .
$$

We have $0 \leq \lambda_{1} \leq \lambda_{2} \leq \cdots \leq \lambda_{\kappa}<\lambda_{\kappa+1}=+\infty$. The polar convex set $(\square-\square)^{*} \subset N_{\mathbb{R}}$ coincides with the set of linear functionals $\varphi \in N_{\mathbb{R}}$ such that length $\varphi(\square) \leq 1$. It is unbounded if and only if $\kappa<\operatorname{rank} M$. The Minkowski successive minima of $\left(N,(\square-\square)^{*}\right)$ are

$$
\lambda_{i}^{*}=\lambda_{i}\left(N,(\square-\square)^{*}\right)=\sup \left\{t \geq 0 ; \operatorname{dim} N \cap t \cdot(\square-\square)^{*}<i\right\} .
$$

Example 4.4. Consider $(X, L)=\left(\mathbb{P}^{d}, \mathcal{O}(w)\right)$, where $w$ is a positive integer. Since the ambient is homogeneous, $\epsilon_{i}(L, x)=\epsilon_{i}(L)$ for all $x \in X$ and $i$. Then

$$
\epsilon_{1}(L)=\cdots=\epsilon_{d}(L)=\sqrt[d]{\operatorname{vol}(L)}=w .
$$

Let $e_{1}, \ldots, e_{d}$ be the standard basis of $\mathbb{Z}^{d}$. The moment polytope of $L$ is

$$
\square=\left\{\sum_{i=1}^{d} x_{i} e_{i} ; x_{i} \geq 0, \sum_{i=1}^{d} x_{i} \leq w\right\}
$$

The difference $\square-\square$ is the convex hull of $\pm w e_{i}(1 \leq i \leq d), \pm w\left(e_{i}-e_{j}\right)(1 \leq i<j \leq d)$, which is contained in $\left\{\sum_{i=1}^{d} x_{i} e_{i} ;\left|x_{i}\right| \leq w,\left|x_{i}+x_{j}\right| \leq w\right\}$. We compute

$$
\lambda_{i}\left(\mathbb{Z}^{d}, \square-\square\right)=\frac{1}{w}(1 \leq i \leq d)
$$

Let $e_{1}^{*}, \ldots, e_{d}^{*}$ be the dual basis of $\check{\mathbb{Z}}^{d}$. The polar body $(\square-\square)^{*}$ is $\left\{\sum_{i=1}^{d} x_{i}^{*} e_{i}^{*} ;\left|x_{i}^{*}\right| \leq 1 / w,\left|x_{i}^{*}-x_{j}^{*}\right| \leq\right.$ $1 / w\}$. We compute

$$
\lambda_{i}\left(\check{\mathbb{Z}}^{d},(\square-\square)^{*}\right)=w(1 \leq i \leq d) .
$$

Example 4.5. Consider $(X, L)=\left(\left(\mathbb{P}^{1}\right)^{d}, \mathcal{O}\left(w_{1}, \ldots, w_{d}\right)\right)$, where $w_{1} \geq w_{2} \geq \cdots \geq w_{d}$ are positive integers. Since the ambient is homogeneous, $\epsilon_{i}(L, x)=\epsilon_{i}(L)$ for all $x$ and $i$. Let $x=[1: 0]^{d} \in X$. Then $\operatorname{Bs}\left|\mathcal{I}_{x}^{t+} L\right|_{\mathbb{Q}}$ consists of the invariant cycles $Y$ through $x$ (affine spaces with coordinates $z_{i}(i \in$ $I)$ ) such that $\sum_{i \in I} w_{i} \leq t$. Therefore $\epsilon_{i}(L)=\min _{|I|=d-i+1} \sum_{j \in I} w_{j}$. Since we ordered the weights, we obtain

$$
\epsilon_{i}(L)=\sum_{j=i}^{d} w_{j}
$$


The volume is

$$
\operatorname{vol}(L)=d ! \prod_{i=1}^{d} w_{i}
$$

The moment polytope of $L$ is $\square=\prod_{i=1}^{d}\left[0, w_{i}\right] \subset \mathbb{R}^{d}$. Then $\square-\square=\prod_{i=1}^{d}\left[-w_{i}, w_{i}\right]$, so

$$
\lambda_{i}\left(\mathbb{Z}^{d}, \square-\square\right)=\frac{1}{w_{i}} .
$$

The polar body $(\square-\square)^{*}$ is $\left\{x \in \check{\mathbb{R}}^{d} ; \sum_{i=1}^{d}\left|x_{i}\right| w_{i} \leq 1\right\}$. Therefore

$$
\lambda_{i}\left(\check{\mathbb{Z}}^{d},(\square-\square)^{*}\right)=w_{d-i+1} \text {. }
$$

We obtain

$$
\frac{\epsilon_{i}(L)}{\lambda_{d-i+1}^{*}}=\epsilon_{i}(L) \cdot \lambda_{i}=\frac{w_{i}+\cdots+w_{d}}{w_{i}} \in[1, d-i+1] .
$$

Lemma 4.6. $\epsilon_{j}(L) \cdot \lambda_{j} \geq 1$.

Proof. Denote $\mu=1 / \lambda_{j}(M, \square-\square)$. There exist $u_{1}, \ldots, u_{j} \in M$, primitive and linearly independent, such that $\mu u_{i}=m_{i}^{\prime}-m_{i}$ for some $m_{i}, m_{i}^{\prime} \in \square$. The inclusion $\left[m_{i}, m_{i}^{\prime}\right] \subset \square$ induces a dominant rational map

$$
\varphi_{i}:(X, L) \rightarrow\left(\mathbb{P}^{1}, \mathcal{O}(\mu)\right), \bar{L} \geq \overline{\mathcal{O}_{\mathbb{P}^{1}}(\mu)} .
$$

Therefore there exists $\mu F_{i}+D_{i} \in|L|_{\mathbb{Q}}$, where $F_{i}$ is the fiber of $\varphi_{i}$ through 1 , and $D_{i}$ is an effective invariant divisor on $X$. We have $F_{i} \cap T_{N}=T_{N \cap u_{i}^{\perp}}$. Since $u_{i}$ are linearly independent, we have $\operatorname{codim}_{1} \cap_{i=1}^{j} F_{i}=j$.

We obtain $\operatorname{codim}_{1} \operatorname{Bs}\left|\mathcal{I}_{1}^{\mu} L\right|_{\mathbb{Q}} \geq j$. Therefore $\mu \leq \epsilon_{j}(L)$.

Lemma 4.7. $\epsilon_{d-j+1}(L) \leq j \cdot \lambda_{j}^{*}$.

Proof. Suppose $\lambda_{j}^{*}=\lambda>0$. There exist $\varphi_{1}, \ldots, \varphi_{j} \in N \cap \lambda \cdot(\square-\square)^{*}$, linearly independent. The property $\varphi_{i} \in \lambda \cdot(\square-\square)^{*}$ means that the interval $\varphi_{i}(\square)$ has length at most $\lambda$. Consider the induced homomorphism of lattices

$$
\left(\varphi_{1}, \ldots, \varphi_{j}\right): M \rightarrow \mathbb{Z}^{j}
$$

whose image has rank $j$ since $\varphi_{i}$ are linearly independent. Then $\square$ is mapped onto a polytope of dimension $j$, contained in $\prod_{i=1}^{j}\left[x_{i}, x_{i}+\lambda\right]$ for some $x_{1}, \ldots, x_{j} \in \mathbb{R}$. The lattice homomorphism induces a dominant rational map $X \rightarrow Y^{d-j}$ whose fiber $\left(F,\left.L\right|_{F}\right)$ through $x=1$ has dimension $j$, and is dominated by $\left(\mathbb{P}^{1}, \mathcal{O}_{\mathbb{P}^{1}}(\lambda)\right)^{j}$. Therefore

$$
\epsilon_{1}\left(\left.L\right|_{F}, 1\right) \leq j \lambda
$$

by Example4.5. Let $D \in\left|\mathcal{I}_{1}^{j \lambda+} L\right|_{\mathbb{Q}}$. Then $\left.D\right|_{F} \in\left|\mathcal{I}_{1}^{j \lambda+}\left(\left.L\right|_{F}\right)\right|_{\mathbb{Q}}$, which is empty since $\epsilon_{1}\left(\left.L\right|_{F}, 1\right) \leq j \lambda$. Therefore $\left.D\right|_{F}=0$. We deduce

$$
F \subseteq \operatorname{Bs}\left|\mathcal{I}_{1}^{j \lambda+} L\right|_{\mathbb{Q}}
$$

Therefore $\operatorname{codim}_{1} \operatorname{Bs}\left|\mathcal{I}_{1}^{j \lambda+} L\right|_{\mathbb{Q}} \leq d-j<d-j+1$. Then $\epsilon_{d-j+1}(L) \leq j \lambda$.

Remark 4.8. For $\epsilon_{d}(L)$, Lemmas 4.6, 4.7 state that $\lambda_{d}^{-1} \leq \epsilon_{d}(L) \leq \lambda_{1}^{*}$. These inequalities also follow from [11, Theorem 3.6].

Theorem 4.9. The invariants $\epsilon_{i}(L), 1 / \lambda_{i}, \lambda_{d-i+1}^{*}$ are all equivalent. More precisely,

$$
1 \leq \epsilon_{i}(L) \cdot \lambda_{i} \leq d \cdot \frac{\epsilon_{i}(L)}{\lambda_{d-i+1}^{*}} \leq d(d-i+1)
$$

Proof. Use Banaszczyk's bound [2] in Mahler's transference theorem $1 \leq \lambda_{i} \lambda_{d-i+1}^{*} \leq d$ for the second inequality, and the two lemmas above. 
Theorem 4.10. The Seshadri constant of $L$ at a very general point is proportional to the lattice width of the moment polytope $\square_{L}$. More precisely,

$$
\frac{\operatorname{width}\left(\square_{L}\right)}{d} \leq \epsilon(L) \leq \operatorname{width}\left(\square_{L}\right)
$$

Proof. If $L$ is not big, both invariants are zero. Suppose $L$ is big. Then $\epsilon(L)=\epsilon_{d}(L)$ and $\operatorname{width}\left(\square_{L}\right)=\lambda_{1}^{*}$, and we can apply the above theorem.

Remark 4.11. For toric varieties, we may establish the inequalities

$$
1 \leq \frac{\operatorname{vol}(L)}{\prod_{i=1}^{d} \epsilon_{i}(L)} \leq d !
$$

of Theorem 3.6 as follows: the left hand side inequality is easy to see. For the right hand side, recall the second main theorem of Minkowski, in the stronger form due to Davenport-Estermann:

$$
\frac{1}{d !} \leq \operatorname{vol}_{M}(\square) \cdot \prod_{i=1}^{d} \lambda_{i}(M, \square-\square) \leq 1 .
$$

We have $\operatorname{vol}(L)=d ! \operatorname{vol}_{M}(\square)$. From $\epsilon_{i} \lambda_{i} \geq 1$, we obtain $\prod_{i} \epsilon_{i} \cdot \prod_{i} \lambda_{i} \geq 1$. Therefore $\operatorname{vol}(L) \leq$ $d ! \prod_{i} \epsilon_{i}$

\section{Adjoint linear systems and the Flatness Theorem of Khinchin}

Recall first results of Demailly [5] and Ein, Küchle, Lazarsfeld [7]:

Theorem 5.1. Let $X$ be a smooth projective variety of dimension d, let $L$ be a nef and big $\mathbb{Q}$-Cartier divisor on $X$ whose fractional part has normal crossing support. Let $\epsilon$ be the Seshadri constant of $L$ at a very general point $x \in X$, which coincides with $\epsilon_{d}(L, x)$. The following properties hold:

1) The jet map $\Gamma\left(\left\lceil K_{X}+L\right\rceil\right) \rightarrow \mathcal{O}_{x} / \mathcal{I}_{x}^{1+\lceil\epsilon-d-1\rceil}$ is surjective. In particular,

$$
\operatorname{dim}_{k} \Gamma\left(\left\lceil K_{X}+L\right\rceil\right) \geq\left(\begin{array}{c}
\lceil\epsilon-1\rceil \\
d
\end{array}\right)=\frac{1}{d !} \prod_{i=1}^{d}\lceil\epsilon-i\rceil .
$$

2) If $\epsilon>d$, then $\Gamma\left(\left\lceil K_{X}+L\right\rceil\right) \neq 0$.

3) If $\epsilon>d+1$, then $\left|\left\lceil K_{X}+L\right\rceil\right|$ maps $X$ onto a variety of dimension $d$.

4) If $\epsilon>2 d$, then $\left|\left\lceil K_{X}+L\right\rceil\right|$ maps $X$ birationally onto a variety of dimension $d$.

Proof. 1) Let $f: Y \rightarrow X$ be the blow-up at $x$, with exceptional divisor $E$. Since $L$ has integer coefficients near $x,\left\lceil K_{Y}+f^{*} L\right\rceil=f^{*}\left\lceil K_{X}+L\right\rceil+(d-1) E$. We may suppose $p=\lceil\epsilon-d-1\rceil$ is non-negative. Then $\left\lceil K_{Y}+f^{*} L-(p+d) E\right\rceil=f^{*}\left\lceil K_{X}+L\right\rceil-(p+1) E$ and

$$
f_{*} \mathcal{O}_{Y}\left(\left\lceil K_{Y}+f^{*} L-(p+d) E\right\rceil\right)=\mathcal{I}_{x}^{p+1}\left(\left\lceil K_{X}+L\right\rceil\right) .
$$

By the Leray spectral sequence, the natural homomorphism

$$
H^{1}\left(X, \mathcal{I}_{x}^{p+1}\left(\left\lceil K_{X}+L\right\rceil\right)\right) \rightarrow H^{1}\left(Y,\left\lceil K_{Y}+f^{*} L-(p+d) E\right\rceil\right)
$$

is injective.

But $f^{*} L-(p+d) E=f^{*} L-(\lceil\epsilon\rceil-1) E$ is nef and big, since $\lceil\epsilon\rceil-1<\epsilon$. By Kawamata-Viehweg vanishing, $H^{1}\left(Y,\left\lceil K_{Y}+f^{*} L-(p+d) E\right\rceil\right)=0$. Therefore $H^{1}\left(X, \mathcal{I}_{x}^{p+1}\left(\left\lceil K_{X}+L\right\rceil\right)\right)=0$. Then $\Gamma\left(\left\lceil K_{X}+L\right\rceil\right) \rightarrow \mathcal{O}_{x} / \mathcal{I}_{x}^{p+1}$ is surjective. Since $x$ is a smooth point, the right hand side has dimension $\left(\begin{array}{c}p+d \\ d\end{array}\right)$.

2) This follows from 1$)$.

3) The 1-jet map $\Gamma\left(\left\lceil K_{X}+L\right\rceil\right) \rightarrow \mathcal{O}_{x} / \mathcal{I}_{x}^{2}$ is surjective. Therefore $\left|\left\lceil K_{X}+L\right\rceil\right|$ moves, and the induced rational map is generically finite. 
4) Let $x, y$ be two very general points, let $f: Y \rightarrow X$ be the blow-up at $x, y$. Then

$$
f^{*} L-d E_{x}-d E_{y}=\frac{1}{2}\left(f^{*} L-2 d E_{x}\right)+\frac{1}{2}\left(f^{*} L-2 d E_{y}\right)
$$

is nef and big. By Kawamata-Viehweg vanishing, $H^{1}\left(Y,\left\lceil K_{Y}+f^{*} L-d E_{x}-d E_{y}\right\rceil\right)=0$. But $\left\lceil K_{Y}+f^{*} L-d E_{x}-d E_{y}\right\rceil=f^{*}\left\lceil K_{X}+L\right\rceil-E_{x}-E_{y}$. Therefore $H^{1}\left(X, \mathcal{I}_{x} \otimes \mathcal{I}_{y}\left(\left\lceil K_{X}+L\right\rceil\right)\right)=0$.

We generalize the flatness theorem of Khinchin. The original statement says that a convex body which contains no lattice points must have lattice width bounded above by a constant which depends only on the dimension (see [12] and part a) of the theorem below). We show that the same conclusion holds if the lattice points of the convex body are degenerate (part b) of the theorem below).

Theorem 5.2. Let $M \simeq \mathbb{Z}^{d}$ be a lattice, let $\square \subset M_{\mathbb{R}}$ be a compact convex set, of dimension d. Let $w$ be the lattice width of $\square$ with respect to $M$.

a) If $w>d^{2}$, then $M \cap \operatorname{int} \square \neq \emptyset$.

b) If $w>d(d+1)$, then $\operatorname{dim}(M \cap \operatorname{int} \square)=d$.

c) If $w>2 d^{2}$, then $M \cap \operatorname{int} \square$ spans $M$.

d) $\sqrt[d]{d !|M \cap \operatorname{int} \square|} \geq \frac{w}{d}-d$.

e) $\sqrt[d]{d ! \operatorname{vol}_{M}(\square)} \geq \frac{w}{d}$

Proof. The width is continuous with respect to the approximation $\lim _{n \rightarrow \infty} \operatorname{Conv}\left(\frac{1}{n} M \cap \square\right)=\square$. Therefore we may suppose $\square$ is the convex hull of finitely many points in $M_{\mathbb{Q}}$.

Let $X$ be a toric desingularization of the projective model of the graded $\operatorname{ring} \oplus_{n \geq 0} \oplus_{m \in M \cap n \square} k \cdot \chi^{m}$. There exists a $\mathbb{Q}$-Cartier divisor $L$ on $X$, semiample and big, supported by the invariant prime divisors of $X$, such that $\square$ is the moment polytope of $L$. We compute

$$
\Gamma\left(\left\lceil K_{X}+L\right\rceil\right)=\oplus_{m \in M \cap i n t} \nabla \cdot \chi^{m} .
$$

Therefore the semi-invariant basis of $\Gamma\left(\left\lceil K_{X}+L\right\rceil\right)$ is in one-to-one correspondence to the interior lattice points of $\square$. Denote $A=M \cap$ int $\square$. The linear system $\left|\left\lceil K_{X}+L\right\rceil\right|$ is non-empty if and only if $A$ is non-empty, it maps $X$ onto a variety of the same dimension if and only if $A-a$ generates the $\mathbb{R}$-vector space $M_{\mathbb{R}}$, for every $a \in A$, and maps $X$ birationally onto a variety of the same dimension if and only if $A-a$ generates the lattice $M$, for every $a \in A$.

Let $\epsilon$ be the Seshadri constant of $L$ at a very general point of $X$. We have $\epsilon=\epsilon_{d}(L)$. By Theorem 4.10, $\epsilon \geq \frac{w}{d}$. Therefore the claims are just a restatement of Theorem 5.1 for $(X, L)$.

Part a) of the theorem was proved by Kannan, Lovász with $d^{2}$ replaced by $c d^{2}$, for some constant c. It is expected that the optimal bound is linear in $d$ (see [12]). Parts b) and c) are new statements, while d) and e) improve similar bounds in [12].

\section{Appendix on Geometry of Numbers}

We recall the definitions and results from the Geometry of Numbers that we use. For proofs and more, the reader may consult [14]. Let $M \simeq \mathbb{Z}^{d}$ be a lattice of rank $d$. The dual lattice $N=\check{M}$ is defined as $\operatorname{Hom}_{\mathbb{Z}}(M, \mathbb{Z})$, and we have a duality pairing $N \times M \rightarrow \mathbb{Z},\langle\varphi, m\rangle=\varphi(m)$.

A subset $A \subseteq M$ spans the lattice $M$ if the difference set $A-A=\left\{a^{\prime}-a ; a^{\prime}, a \in A\right\}$ generates the lattice $M$. The dimension of a subset $A \subseteq M_{\mathbb{R}}$, denoted $\operatorname{dim}(A)$, is the dimension of the $\mathbb{R}$ vector space generated by $A-A$. The $\mathbb{Q}$-dimension of a subset $A \subseteq M_{\mathbb{R}}$, denoted $\operatorname{dim}_{\mathbb{Q}}(A)$, is the dimension of the $\mathbb{Q}$-vector space generated by $A \cap M_{\mathbb{Q}}-A \cap M_{\mathbb{Q}}$. We have $\operatorname{dim}_{\mathbb{Q}}(A) \leq \operatorname{dim}(A)$, and equality holds if $A \subseteq M_{\mathbb{Q}}$. If $\square \subseteq M_{\mathbb{R}}$ is a convex set, then $\operatorname{dim}_{\mathbb{Q}}(\square)=d$ if and only if $\operatorname{dim}(\square)=d$. 
For a convex set $\square \subseteq M_{\mathbb{R}}$, the polar convex set $\square^{*} \subseteq N_{\mathbb{R}}$ is defined as

$$
\square^{*}=\left\{\varphi \in N_{\mathbb{R}}^{*} ;\langle\varphi, m\rangle \geq-1 \forall m \in \square\right\} .
$$

To $\square$ we can associate the difference convex set $\square-\square=\left\{m^{\prime}-m ; m^{\prime}, m \in \square\right\}$, which is 0-symmetric. The polar convex set $(\square-\square)^{*}$ consists of the linear functionals $\varphi \in N_{\mathbb{R}}$ such that the interval $\varphi(\square)$ has length at most 1.

The lattice width of a compact convex set $\square \subset M_{\mathbb{R}}$ is defined as the smallest length of any interval $\varphi(\square)$, with all $\varphi \in N \backslash 0$. It coincides with the first minimum of Minkowski of $(\square-\square)^{*}$ with respect to $N$.

Let $\square \subseteq M_{\mathbb{R}}$ be a closed convex set which contains the origin. For $i \geq 1$, the $i$-th successive minimum of $(M, \square)$ is defined by

$$
\lambda_{i}(M, \square)=\sup \{t \geq 0 ; \operatorname{dim}(M \cap t \square)<i\} .
$$

We obtain an increasing chain $0 \leq \lambda_{1} \leq \lambda_{2} \leq \cdots \leq \lambda_{d+1}=+\infty$. Note that $\lambda_{i}$ is a finite real number if and only if $i \leq \operatorname{dim}_{\mathbb{Q}}(\square)$, and $\lambda_{i}(M, c \square)=\lambda_{i}(M, \square) / c$ for every $c>0$. In case $\operatorname{dim}(\square)=d$, we have the equivalent (Minkowski's) definition

$$
\lambda_{i}(M, \square)=\inf \{t \geq 0 ; \operatorname{dim}(M \cap t \square) \geq i\}
$$

The second main theorem of Minkowski, in the generalized form due to Davenport-Estermann, is

Theorem 6.1. Let $\square \subset M_{\mathbb{R}}$ be a convex set of dimension $d$. Then

$$
\frac{1}{d !} \leq \operatorname{vol}_{M}(\square) \cdot \prod_{i=1}^{d} \lambda_{i}(M, \square-\square) \leq 1 .
$$

The transference theorem of Mahler, with the improved upper bound due to Banaszczyck [2, Theorem 2.1], is

Theorem 6.2. Let $\square \subset M_{\mathbb{R}}$ be a 0-symmetric compact convex set of dimension d. Let $\square^{*} \subset N_{\mathbb{R}}$ be the polar body. Then

$$
1 \leq \lambda_{i}(M, \square) \cdot \lambda_{d-i+1}\left(N, \square^{*}\right) \leq d(1 \leq i \leq d)
$$

With the original upper bound of Mahler $\left((d !)^{2}\right.$ instead of $\left.d\right)$, the transference theorem can be deduced from the second main theorem of Minkowski. The upper bound $d$ is sharp in dimension one. In dimension two, Banaszczyck [2, Proof of Theorem 2.1] mentions the upper bound $2 / \sqrt{3}$. We show next that the sharp upper bound in dimension two is in fact $3 / 2$.

Sharp transference theorem in dimension two. Let $\square \subset \mathbb{R}^{2}$ be a 0 -symmetric polytope, of dimension 2, with $\lambda_{1}\left(\mathbb{Z}^{2}, \square\right)=1$. This means that $\mathbb{Z}^{2} \cap$ int $\square=\{0\}$ and $\mathbb{Z}^{2} \cap \partial \square \neq \emptyset$.

We say that $\square$ is maximal if every top face of $\square$ contains a lattice point in its relative interior. We bring $\square$ into maximal position, preserving the initial hypothesis, as follows. Since $\square$ is 0 -symmetric, the top faces come in pairs. Fix a pair $F,-F$. We slide out these top faces until they either a) hit a lattice point in their relative interior, or b) these faces collapse to a point (they become redundant). Apriori, it is also possible that we may slide out the faces to infinity, but one may easily check that this is impossible. We repeat the argument for all pairs of top faces.

In finitely many steps, we enlarged $\square \subset \square^{\prime}$ such that $\square^{\prime} \subset \mathbb{R}^{2}$ is a 0 -symmetric polytope, of dimension 2, with $\lambda_{1}\left(\mathbb{Z}^{2}, \square^{\prime}\right)=1$, and $\square^{\prime}$ is maximal. It is enough to prove transference for $\square^{\prime}$.

From now on, we suppose $\square$ is maximal.

Lemma 6.3. On each top face $F$ of $\square$ choose exactly one relative interior lattice point $u_{F}$, in such a way so that $-u_{F}$ is the choice for the opposite face $-F$. Let $Q$ be the convex hull of these lattice points. Then, after possibly changing the basis of $\mathbb{Z}^{2}, Q$ is one of the following: 
1) The square with vertices $(1,0),(0,1),(-1,0),(0,-1)$.

2) The polytope with vertices $(1,0),(0,1),(-1,1),(-1,0),(0,-1),(1,-1)$.

Proof. Let $u_{1}, u_{2}$ be two adjacent vertices of $Q$. Then $\operatorname{Conv}\left(0, u_{1}, u_{2}\right) \backslash\left\{u_{1}, u_{2}\right\}$ is contained in int $\square$. Therefore $\operatorname{Conv}\left(0, u_{1}, u_{2}\right)$ contains no other lattice points besides its vertices. Therefore $u_{1}, u_{2}$ is a basis of $\mathbb{Z}^{2}$. We may suppose $u_{1}=(1,0)$ and $u_{2}=(0,1)$. Thus $u_{1} u_{2}$ is a top face of $Q$. The opposite is also a top face. The other top faces are contained in the second and fourth quadrant. Enough to look inside the second quadrant.

If $Q$ has 4 vertices, we obtain 1). Suppose $Q$ has at least 6 vertices. Then there are at least two top faces in the second quadrant. But $(0,1)$ and $(-1,0)$ are vertices of $Q$, and $(-1,1)$ is not contained in the interior of $Q$. Therefore $Q$ has exactly one vertex, namely $(-1,1)$, in the interior of the second quadrant. We are in case 2).

Case 1). Here $\square=\cap_{i=1}^{2}\left\{(x, y) \in \mathbb{R}^{2} ;\left|\left\langle u_{i},(x, y)\right\rangle\right| \leq 1\right\}$, where $u_{1}=\left(1, \beta_{1}\right), u_{2}=\left(-\beta_{2}, 1\right)$ and $\beta_{1}, \beta_{2} \in[0,1)$. Indeed, we may suppose $(1,1)$ is separated from $\square$ by the edge passing through $(1,0)$. The exterior normal to this edge is $u_{1}=\left(1, \beta_{1}\right)$ for some $\beta_{1} \geq 0$. Since $\left\langle \pm u_{1},(0,1)\right\rangle<1$, we deduce $\beta_{1}<1$. The lattice point $(-1,1)$ must be separated from $\square$ by its edge through $(0,1)$. The exterior normal to this edge is $u_{2}=\left(-\beta_{2}, 1\right)$ for some $\beta_{2} \geq 0$. Since $\left\langle \pm u_{1},(1,0)\right\rangle<1$, we deduce $\beta_{1}<1$. Conversely, any polytope $\square$ as above satisfies $\mathbb{Z}^{2} \cap$ int $\square=\{0\}$.

Since $u_{1}, u_{2}$ are linearly independent, we compute

$$
\square^{*}=\left\{\alpha_{1} u_{1}+\alpha_{2} u_{2} ;\left|\alpha_{1}\right|+\left|\alpha_{2}\right| \leq 1\right\}=\operatorname{Conv}\left( \pm u_{1}, \pm u_{2}\right) .
$$

Let $z=\left(z_{1}, z_{2}\right) \in \check{\mathbb{Z}}^{2}$. Then

and $z \in h(z) \cdot \square^{*}$, where

$$
z=\frac{z_{1}+\beta_{2} z_{2}}{1+\beta_{1} \beta_{2}} u_{1}+\frac{z_{2}-\beta_{1} z_{1}}{1+\beta_{1} \beta_{2}} u_{2}
$$

We compute

$$
h(z)=\frac{\left|z_{1}+\beta_{2} z_{2}\right|}{1+\beta_{1} \beta_{2}}+\frac{\left|z_{2}-\beta_{1} z_{1}\right|}{1+\beta_{1} \beta_{2}} .
$$

$$
h(1,0)=\frac{1+\beta_{1}}{1+\beta_{1} \beta_{2}}, h(0,1)=\frac{1+\beta_{2}}{1+\beta_{1} \beta_{2}}, h(1,1)=\frac{2+\beta_{2}-\beta_{1}}{1+\beta_{1} \beta_{2}}, h(-1,1)=\frac{2+\beta_{1}-\beta_{2}}{1+\beta_{1} \beta_{2}} .
$$

We claim that $\lambda_{2}\left(\check{\mathbb{Z}}^{2}, \square^{*}\right) \leq \frac{3}{2}$. Indeed, suppose $0 \leq \beta_{1} \leq \beta_{2}<1$. Then

$$
1+\beta_{1} \leq 1+\beta_{2}, 2+\beta_{1}-\beta_{2} \text { and } 1+\beta_{2}, 2+\beta_{1}-\beta_{2} \leq 2-\beta_{1}+\beta_{2} .
$$

Therefore

$$
\lambda_{2}\left(\check{\mathbb{Z}}^{2}, K^{*}\right) \leq \min \left(\frac{1+\beta_{2}}{1+\beta_{1} \beta_{2}}, \frac{2+\beta_{1}-\beta_{2}}{1+\beta_{1} \beta_{2}}\right) .
$$

One may check that the right hand side is at most $\frac{3}{2}$, with equality only for $\beta_{1}=0, \beta_{2}=\frac{1}{2}$.

Case 2). Here $\square=\cap_{i=1}^{3}\left\{(x, y) \in \mathbb{R}^{2} ;\left|\left\langle u_{i},(x, y)\right\rangle\right| \leq 1\right\}$, where $u_{1}=\left(1, \beta_{1}\right), u_{2}=\left(\beta_{2}, 1\right), u_{3}=$ $\left(-\beta_{3}, 1-\beta_{3}\right)$, and $\beta_{1}, \beta_{2}, \beta_{3} \in(0,1)$. Indeed, we may suppose $(1,1)$ is separated from $\square$ by its edge through $(1,0)$. Let $u_{1}=\left(1, \beta_{1}\right)$ be the exterior normal, so that $\beta_{1} \geq 0$. We have $\left\langle u_{1},(0,1)\right\rangle<1$ and $\left\langle u_{1},(1,-1)\right\rangle<1$. That is $0<\beta_{1}<1$. Let $u_{2}$ and $u_{3}$ be the normalized exterior normals to $Q$ through $(0,1)$ and $(-1,1)$, respectively. We have $\left\langle u_{2},(1,0)\right\rangle<1$ and $\left\langle u_{1},(-1,1)\right\rangle<1$, that is $0<\beta_{2}<1$. Similarly, $\left\langle u_{3},(0,1)\right\rangle<1$ and $\left\langle u_{3},(-1,0)\right\rangle<1$, that is $0<\beta_{3}<1$.

Conversely, any polytope $\square$ as above satisfies $\mathbb{Z}^{2} \cap$ int $\square=\{0\}$. Indeed, once $u_{1}$ is chosen, it is enough to consider the lattice points in the strip $\left|\left\langle u_{1}, \cdot\right\rangle\right| \leq 1$. By symmetry, we may take $y>0$. The lattice points in the strip with $x \leq-1$ must be separated from $\square$ by its top face through $(-1,1)$. We are left with the lattice points $(0, n)(n=1,2,3, \ldots)$. But these are separated from $\square$ by its edge through $(0,1)$. 
Note that $\square^{*}$ is the 0 -symmetric polytope with vertices $\pm u_{1}, \pm u_{2}, \pm u_{3}$, and is inscribed in the polytope $Q^{*}=\operatorname{Conv}( \pm(1,0), \pm(1,1), \pm(0,1), \pm(-1,1))$. For $e \in \check{\mathbb{Z}}^{2} \backslash 0$, let $h(e)=\inf \{t>0 ; e \in$ $\left.t \cdot \square^{*}\right\}$. Denote $s_{e}=\left(1-h(e)^{-1}\right)^{-1}$. We compute

$$
s_{1,0}=\frac{1}{\beta_{2}}+\frac{1}{\beta_{3}}, s_{1,1}=\frac{1}{1-\beta_{1}}+\frac{1}{1-\beta_{2}}, s_{0,1}=\frac{1}{\beta_{1}}+\frac{1}{1-\beta_{3}} .
$$

For $t_{1}, t_{2}>0$, we have $\frac{1}{t_{1}}+\frac{1}{t_{2}} \geq \frac{4}{t_{1}+t_{2}}$. Thus

$$
s_{1,0}+s_{1,1}=\frac{1}{\beta_{2}}+\frac{1}{1-\beta_{2}}+\frac{1}{1-\beta_{1}}+\frac{1}{\beta_{3}} \geq 4+\frac{1}{1-\beta_{1}}+\frac{1}{\beta_{3}}>6 .
$$

Similarly, $s_{1,0}+s_{0,1}>6$ and $s_{1,1}+s_{0,1}>6$. Therefore two of the $s_{1,0}, s_{1,1}, s_{0,1}$ are strictly larger than 3. Therefore $\lambda_{2}\left(\check{\mathbb{Z}}^{2}, \square^{*}\right)<\frac{3}{2}$. We obtained:

Theorem 6.4. Let $\square \subset \mathbb{R}^{2}$ be a 0-symmetric convex body. Then

$$
1 \leq \lambda_{1}\left(\mathbb{Z}^{2}, \square\right) \cdot \lambda_{2}\left(\check{\mathbb{Z}}^{2}, \square^{*}\right) \leq \frac{3}{2}
$$

Proof. The first inequality is trivial. For the second, suppose by contradiction that it fails. Then we may approximate $\square$ with a polytope, and suppose $\square$ is a polytope. If $\square$ is a polytope, we may scale so that $\lambda_{1}=1$, and we are done by the two cases above.

Example 6.5. The upper bound $\frac{3}{2}$ is attained for $\square$ in Case 1 ) with $\beta_{1}=0, \beta_{2}=\frac{1}{2}$. In this case, $\square=\operatorname{Conv}\left( \pm\left(1, \frac{3}{2}\right), \pm\left(1, \frac{1}{2}\right)\right), \square^{*}=\operatorname{Conv}\left( \pm(1,0), \pm\left(-\frac{1}{2}, 1\right)\right)$ and $\lambda_{1}\left(\mathbb{Z}^{2}, \square\right)=1, \lambda_{2}\left(\check{\mathbb{Z}}^{2}, \square^{*}\right)=\frac{3}{2}$.

\section{REFERENCES}

[1] Ambro, F., Variation of log canonical thresholds in linear systems. IMRN 14(1) (2016), 4418 - 4448.

[2] Banaszczyk, W., New bounds in some transference theorems in the geometry of numbers. Math. Ann 296 (1993), $625-635$.

[3] Bauer, T.;Di Rocco, S.;Harbourne, B.; Kapustka, M.;Knutsen, A.;Syzdek, W.; T.Szemberg, T., A primer on Seshadri constants Interactions of classical and numerical algebraic geometry, Contemp. Math. 496, American Mathematical Society, Providence (2009), 33-70.

[4] Birkar, C., The augmented base locus of real divisors over arbitrary fields. Math. Ann 368 (2017), no. 3-4, 905-921.

[5] Demailly, J.P., Singular hermitian metrics on positive line bundles. In: Hulek K., Peternell T., Schneider M., Schreyer FO. (eds) Complex Algebraic Varieties. Lecture Notes in Mathematics, vol 1507 (1992). Springer, Berlin, Heidelberg.

[6] Eckl T., Lower bounds for Seshadri constants. Math. Nachr. 281 (2008), no. 8, 1119-1128.

[7] Ein, L.; Küchle, O.; Lazarsfeld, R., Local positivity of ample line bundles. J. Differential Geom. 42 (2) (1995), $193-219$.

[8] Ein, L.; Lazarsfeld, R.; Mustaţă, M.; Nakamaye, M.; Popa, M., Restricted Volumes and Base Loci of Linear Series. Amer. J. of Math. 131(3) (2009), $607-651$.

[9] Fujita, T., Semipositive line bundles. J. Fac. Sci. Univ. Tokyo Sect. IA Math. 30(2) (1983), 353 - 378.

[10] Fulton, W., Intersection theory, Second edition. Ergebnisse der Mathematik und ihrer Grenzgebiete. SpringerVerlag, Berlin, 1998.

[11] Ito, A., Seshadri constants via toric degenerations. Journal für die reine und angewandte Mathematik 695 $2014,151-174$.

[12] Kannan, R.; Lovász, L., Covering minima and lattice-point-free convex bodies. Ann. of Math. (2) 128 (1988), no. 3, 577-602.

[13] Lazarsfeld, R., Positivity in algebraic geometry I. Ergebnisse der Mathematik und ihrer Grenzgebiete, vol. 48. Springer, Berlin (2004).

[14] Lekkerkerker, C. G., Geometry of numbers. Bibliotheca Mathematica, Vol. VIII Wolters-Noordhoff Publishing, Groningen; North-Holland Publishing Co., Amsterdam-London 1969.

[15] Nakamaye, M., Stable base loci of linear series. Math. Ann. 318 (2000), no. 4, 837-847.

[16] Nakamaye, M., Base loci of linear series are numerically determined. Trans. Amer. Math. Soc. 355(2) (2003), $551-566$. 
[17] Nakamaye, M., Seshadri constants and the geometry of surfaces. J. Reine Angew. Math. 564 (2003), 205 214.

[18] Nakamaye, M., Seshadri constants at very general points. Trans. AMS 357 (8) (2005), 3285 - 3297.

[19] Oda, T., Convex bodies and algebraic geometry. An introduction to the theory of toric varieties. Translated from the Japanese. Ergebnisse der Mathematik und ihrer Grenzgebiete (3), 15. Springer-Verlag, Berlin, 1988. viii +212

Institute of Mathematics "Simion Stollow" of the Romanian Academy, P.O. BOX 1-764, RO014700 Bucharest, Romania.

E-mail address: florin.ambro@imar.ro

Graduate School of Mathematics, Nagoya University, Nagoya, 464-8602, Japan.

E-mail address: atsushi.ito@math.nagoya-u.ac.jp 